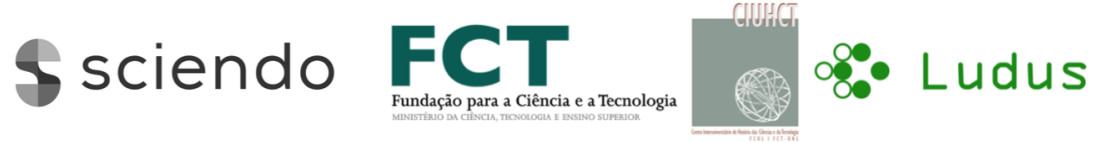

\section{An ALternative ALGORITHM FOR THE $n$-QueEnS PUZZLE}

\author{
David Luque Sacaluga \\ Chiclana de la Frontera 11130 Cádiz, Spain \\ sanchezbereadavid@gmail.com
}

\begin{abstract}
In this paper a new method for solving the problem of placing $n$ queens on a $n \times n$ chessboard such that no two queens directly threaten one another and considering that several immovable queens are already occupying established positions on the board is presented. At first, it is applied to the 8-Queens puzzle on a classical chessboard and finally to the $n$ Queens completion puzzle. Furthermore, this method allows finding repetitive patterns of solutions for any $n$.
\end{abstract}

Keywords: Chessboard; $n$-Queens puzzle; NP-completeness; Patterns

This article is organized in four sections. The introduction reminds the 8-Queens puzzle in a colloquial way, what kind of algorithms are used for solving it and what is the difference between the 8-Queens puzzle and the 8-Queens completion problem. Also, it is clarified that the paper is not related to the $\mathrm{P}$ vs NP Millennium Prize Problem.

A second section introduces the concept of subpanels when a chessboard is broken down. A limited number of queens allowed in every subpanel are defined. An equation representing the several cases is defined and a graphical example solution is shown.

The third section is an analysis of the method applied for any value of $n$. It takes into account if $n$ is even or odd. The different cases depend on the number of queens in the first and last subpanels and the subcases depend on the number of queens to be distributed in the central subpanels. The several alternatives will depend on the position of the queens into subpanels. A graphical representation for helping to understand every case is included. In this point we observe that when $n$ is increased then the number of cases, subcases and alternatives increase too, but when the number of queens preplaced is increased then the number of alternatives, subcases and cases decrease. We can conclude that the problem becomes easiest when the number of queens preplaced is increased. At the end of this section, some pattern rules to extend solutions from smallest to largest 
values of $n$ are explained with two simple examples. We invite to the reader for finding patterns rules from other solutions of the 8-Queens puzzle.

Finally, the appendix is a "time travel to 1850." We don't have a computer and Someone is asking to find all solutions with two queens preplaced at position b4 and d5. Fortunately, we have travelled with a complete analysis of the completion problem in our knapsack.

\section{Introduction}

In September 2017, a lot of news regarding how to win one million dollars appeared on the internet. The reason was a paper published about the complexity of the $n$-Queens Completion Problem by researchers at the University of St. Andrews GJN17. Obviously, curiosity led many uninitiated people to these complex problems (like me) trying to solve it. It was about the millennium problem: P = NP? [CMA21, Coo71, but focusing on a chessboard.

In September 1848, a long time before, Max Bezzel published the 8-Queens puzzle and two years later, Frank Nauck found the first solutions. The method used by Nauck begun by placing a queen on the first column and placing the others queens on the lowest unguarded squares [Sch15], similar to brute force and backtracking techniques used today by programmers but in a time when there were no computers. Brute force is useful for small domains, due to large overheads in sophisticated approaches. Backtracking is an improvement of the brute force approach, which systematically searches for a solution to a problem among all available options; i.e. backtracking checks each possibility until they find the right one. It is a depth-first search of the whole of possible solutions. During the searching, if an alternative is not allowed then it backtracks to the place which presented several alternatives, and tries the next one. If the alternatives are exhausted, then it goes back to the previous choice point and tries the next alternative. If there are no more options, then the search fails [BSR08, BSR10, ACP68.

Back to 1850, Nauck introduced the $n$-Queens Completion puzzle presenting a variant of the 8-Queens puzzle in which some queens were already placed on the chessboard and he asked how to place the rest of the queens [ACJ18].

On one hand, the $n$-Queens puzzle is the problem to place $n$ queens on an $n$ by $n$ chessboard in such a way that none of the queens is attacking another queen and on the other hand, the $n$-Queens Completion puzzle is the problem to place $k<n$ queens on a $n$ by $n$ chessboard in which $(n-k)$ queens are already placed.

Let's try to solve the 8-Queens puzzle. Can you place eight queens on a chessboard in such a way that none of the queens is attacking another queen? It's not so hard, is it? You can try it and finally you will get a solution. To check that a given solution is correct is easy because once the queens are placed, only one queen is allowed in every row, column or diagonal. But, can you find all the solutions? Why not? If the chessboard 
is finite then it is only a patient and time question. What about when $n$ is increased? You will have more solutions and you will need more time to find them... but if you have got a computer and a good algorithm then the problem is not so hard. Currently, the algorithms used for solving this kind of problems are very efficient when the number of queens is moderated but not too efficient with "monster" values of $n$. Up to date, the $27 \times 27$ chessboard is the higher value calculated with $234,907,967,154,122,528$ solutions $[?]$.

There are several strategies for solving the $n$-Queens puzzle [AY89, [BS09], BD75, [BM02, Eng07], ET92, [Ber91]. For instance, there is a simple way to extend a solution for dimension $(n-1) \times(n-1)$ to dimension $n \times n$. Figure 1 shows a solution that exhibits stair-stepped patterns for $n=8$ to $n=9$ by adding a queen in a corner square of the chessboard.
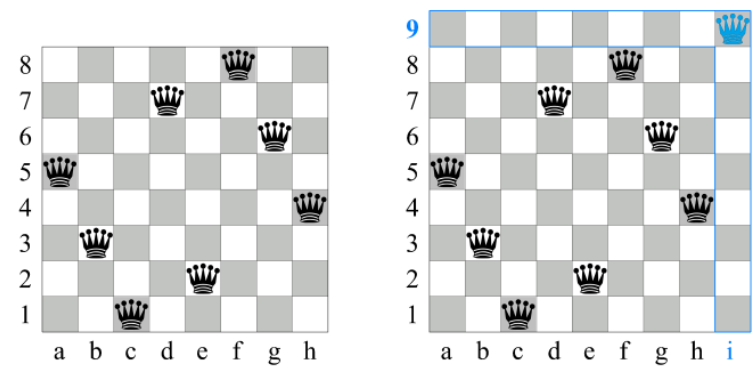

Figure 1: Example of extended solution for dimension $n=8$ to $n=9$

The problem becomes more difficult when several immovable queens are already occupying established positions on the board, that is, "checking" becomes easier because there are fewer options, but "solving" becomes more difficult because the immovable queens preplaced don't ensure the existence of a solution. For instance, Figure 2 shows two queens preplaced on the chessboard. Can you place six queens? Now, no solution is allowed.

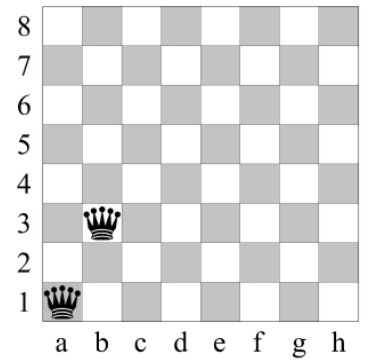

Figure 2: Example of 8-Queens completion puzzle.

The "alternative" algorithm presented in this paper could be a "sophisticated" back- 
tracking algorithm to be used for speeding up the process because processing several cases in any order and try the most constrained ones first to obtain a solution from a current partial solution is allowed. Anyway, it is just an algorithm without the claim that it works in polynomial time and it does not concern the $\mathrm{P}=\mathrm{NP}$ problem. Nevertheless, if you find an algorithm to determine the existence of a solution of the $n$-Queens completion puzzle, it could be applied for solving other NP-complete problems [GJ79], $[?]$.

\section{Method description on the 8-Queens puzzle}

The method is based on breaking down a chessboard in several subpanels. Considering the game's rule that not two queens can share the same row, column, or diagonal, a minimum and maximum number of queens allowed on every subpanel is defined. From these data, an equation and a list of possible cases and subcases are defined. Depending on the number and the position of queens on every subpanel, the reconstruction of the chessboard can be performed. Solution appears when constraints are satisfied.

A 64-square chessboard can be broken down into four subpanels as shown in Figure 3.
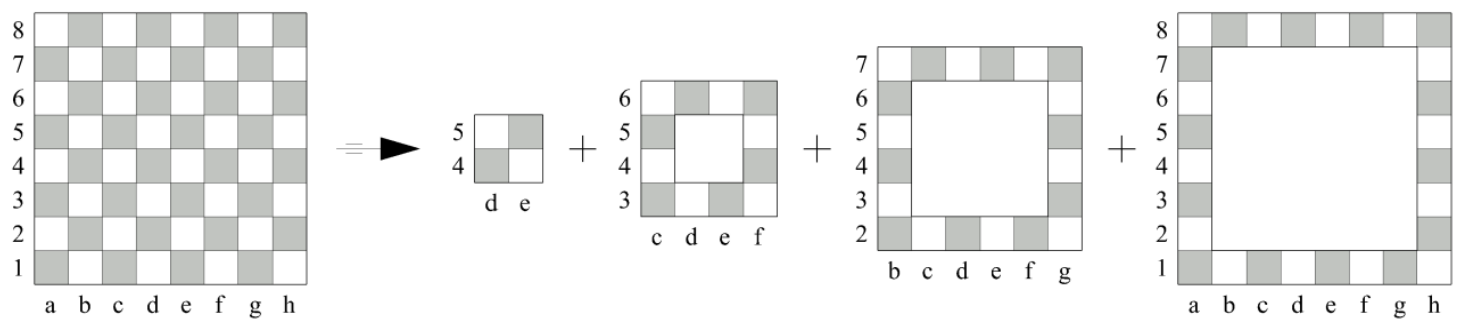

Figure 3: Breaking down of a chessboard in four subpanels.

Let be $S^{4}$ the subpanel shaped by d4, d5, e4 and e 5 squares. A minimum of zero queens and a maximum of one queen for placing on subpanel $S^{4}$ will be allowed.
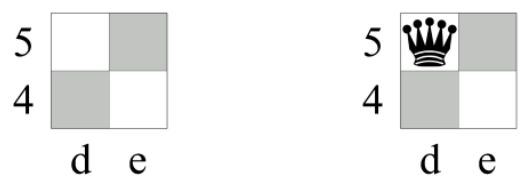

Figure 4: Example of minimum and maximum number of queens allowed on subpanel $S^{4}$.

Let be $S^{12}$ the subpanel shaped by c3, c4, c5, c6, d3, d6, e3, e6, f3, f4, f5 and f6 squares. A minimum of zero queens and a maximum of four queens for placing on subpanels $S^{12}$ will be allowed. 

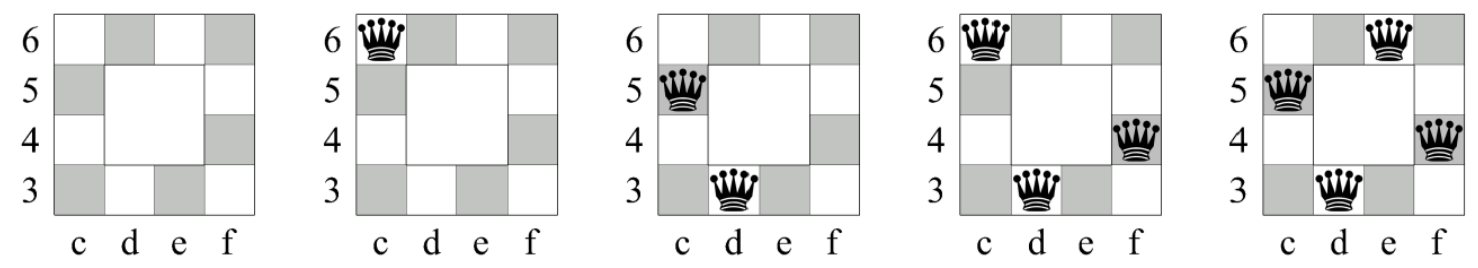

Figure 5: Example of minimum and maximum number of queens allowed on subpanels $S^{12}$.

Let be $S^{20}$ the subpanel shaped by b2, b3, b4, b5, b6, b7, c2, c7, d2, d7, e2, e7, f2, $\mathrm{f} 7, \mathrm{~g} 2, \mathrm{~g} 3, \mathrm{~g} 4, \mathrm{~g} 5, \mathrm{~g} 6$ and $\mathrm{g} 7$ squares. A minimum of zero queens and a maximum of four queens for placing on subpanels $S^{20}$ will be allowed.
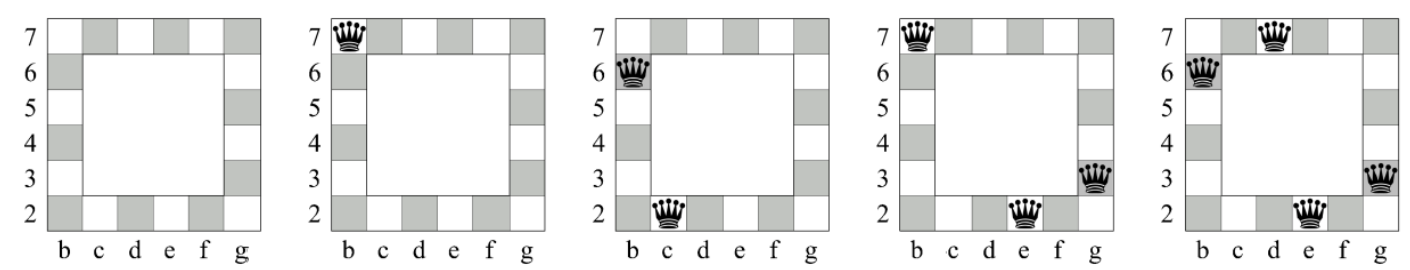

Figure 6: Example of minimum and maximum number of queens allowed on subpanels $S^{20}$.

Let be $S^{28}$ the subpanel shaped by a1, a2, a3, a4, a5, a6, a7, a8, b1, b8, c1, c8, d1, d8, e1, e8, f1, f8, g1, g8, h1, h2, h3, h4, h5, h6, h7 and h8 squares. A minimum of three queens and a maximum of four queens for placing on subpanel $S^{28}$ will be allowed.
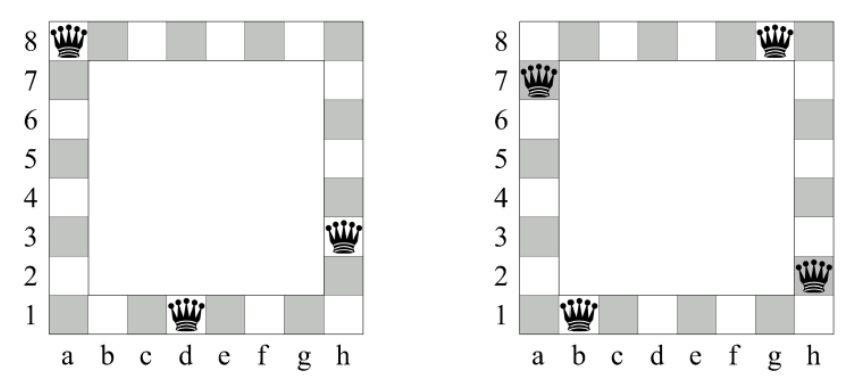

Figure 7: Example of minimum and maximum number of queens allowed on subpanel $S^{28}$

Let be $T^{64}$ a chessboard shaped by 64 squares. Namely $T^{64}$ is shaped by $S^{4}, S^{12}, S^{20}$ and $S^{28}$ subpanels. Considering the eight queens to be placed and the number of queens 
allowed on every subpanel, the several cases allowed for the 8-Queens puzzle are defined in Equation (1).

$$
T^{64}\{8\}=S^{4}\left\{\begin{array}{l}
1 \\
0
\end{array}+S^{12}\left\{\begin{array}{l}
4 \\
3 \\
2 \\
1 \\
0
\end{array}+S^{20}\left\{\begin{array}{l}
4 \\
3 \\
2 \\
1 \\
0
\end{array}+S^{28}\left\{\begin{array}{l}
4 \\
3
\end{array}\right.\right.\right.\right.
$$

In any case, the total number of queens on the chessboard must be equal to eight and the reconstruction of the chessboard depends on the position of the queens at every subpanel. An example is showed in Figure 8.
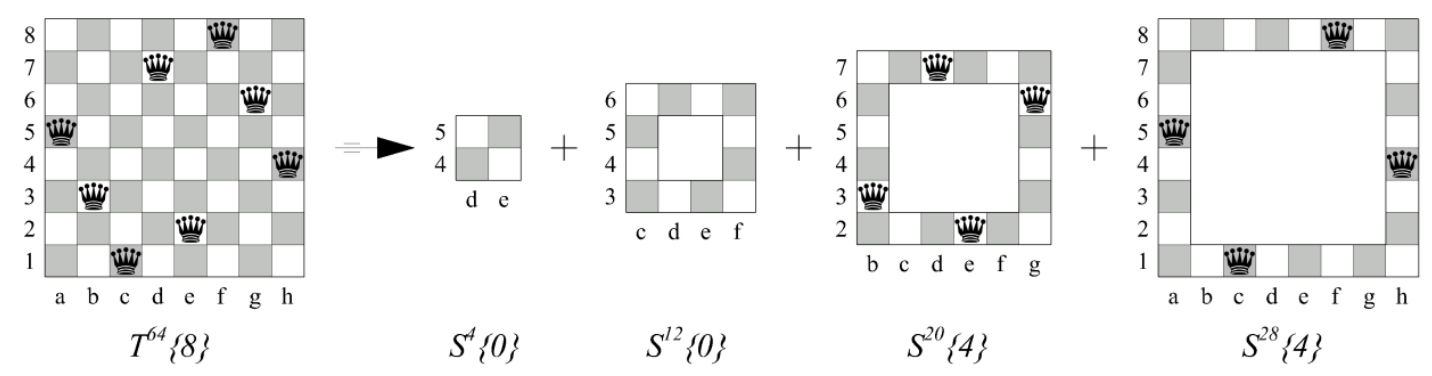

Figure 8: Example of breaking down graphical representation of an 8-Queens puzzle solution.

\section{Method description on a $n \times n$ chessboard}

The method can be used for solving the question about how to place $n$ queens on a $n \times n$ chessboard considering that several immovable queens are already occupying established positions on the board, but it depends on $n$ is even or odd. Also, some pattern rules can be applied from lower values to higher values of $n$.

\subsection{When $n$ is even}

Let $n$ be an even integer number. The number of squares on a $n \times n$ chessboard is equal to the sum of the number of squares on every subpanel:

$$
n^{2}=4+12+20+28+\cdots+n^{2}-(n-2)^{2}
$$

Let $T^{n x n}$ be a chessboard with $n \times n$ squares and let $S^{4}, S^{12}, S^{20}, S^{28}, \ldots, S^{n^{2}-(n-2)^{2}}$ be the subpanels. If we assume $n>2$, the several cases considering the number of queens 
allowed on every subpanel is defined in Equation (2).

$$
T^{n x n}\{n\}=S^{4}\left\{\begin{array}{l}
1 \\
0
\end{array}+S^{12}\left\{\begin{array}{l}
4 \\
3 \\
2 \\
1 \\
0
\end{array}+S^{20}\left\{\begin{array}{l}
4 \\
3 \\
2 \\
1 \\
0
\end{array}+S^{28}\left\{\begin{array} { l } 
{ 4 } \\
{ 3 } \\
{ 2 + S ^ { 3 6 } } \\
{ 1 } \\
{ 0 }
\end{array} \left\{\begin{array}{l}
4 \\
3 \\
2+\cdots+S^{n^{2}-(n-2)^{2}} \\
1 \\
0 \\
3
\end{array}\right.\right.\right.\right.\right.
$$

Equation (2) can be expressed as the Equation (3).

$$
T^{n x n}\{n\}=S^{4}\left\{\begin{array}{l}
1 \\
0
\end{array}+\sum_{i=1}^{\frac{n-4}{2}} S^{12+8(i-1)}\left\{\begin{array}{l}
4 \\
3 \\
2 \\
1 \\
0
\end{array}\right.\right.
$$

In any case, the total number of queens in the subpanels is equal to $n$. Since the subpanels $S^{4}$ and $S^{n^{2}-(n-2)^{2}}$ have less choices, we will split the analysis into four cases depending on the number of queens in these subpanels. They are defined in the Table 1.

\begin{tabular}{|l|c|c|c|c|}
\hline Cases & $T^{n \times n}$ & $S^{4}$ & $\sum_{i=1}^{\frac{n-4}{2}} S^{12+8(i-1)}$ & $S^{n^{2}-(n-2)^{2}}$ \\
\hline Case 1 & $n$ & $\mathbf{1}$ & $n-5$ & $\mathbf{4}$ \\
\hline Case 2 & $n$ & $\mathbf{0}$ & $n-4$ & $\mathbf{4}$ \\
\hline Case 3 & $n$ & $\mathbf{1}$ & $n-4$ & $\mathbf{3}$ \\
\hline Case 4 & $n$ & $\mathbf{0}$ & $n-3$ & $\mathbf{3}$ \\
\hline
\end{tabular}

Table 1: List of four possible cases when $n$ is even.

Depending on the number of queens allowed on the subpanels $\mathrm{S}^{12+8(\mathrm{i}-1)}$, there are several possible subcases. It is represented in Equation 4.

$$
\sum_{i=1}^{\frac{n-4}{2}} S^{12+8(i-1)}=\left\{\begin{array}{l}
\text { Case } 1: n-5 \\
\text { Case } 2: n-4 \\
\text { Case } 3: n-4 \\
\text { Case } 4: n-3
\end{array}\right.
$$

Now we proceed to analyze each case one by one. 
Case 1: The following constraints must be satisfied:

- There is one queen on subpanel $S^{4}$.

- There are four queens on subpanel $S^{n^{2}-(n-2)^{2}}$. So, it is not allowed to place any queen in the corner squares of this subpanel.

- The number of queens to be distributed on the subpanels $S^{12+8(i-1)}$ is $n-5$.

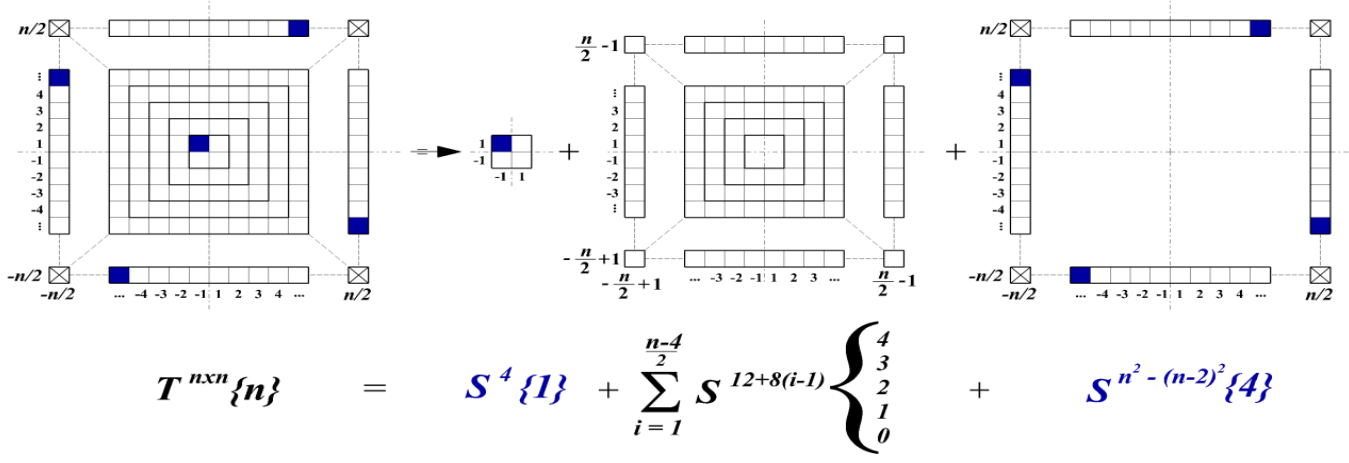

Figure 9: Case 1 when $n$ is even. Example of graphical representation.

Case 2: The following constraints must be satisfied:

- There is not any queen on subpanel $S^{4}$.

- There are four queens on subpanel $S^{n^{2}-(n-2)^{2}}$. So, it is not allowed to place any queen in the corner squares of this subpanel.

- The number of queens to be distributed on the subpanels $S^{12+8(i-1)}$ is $n-4$.

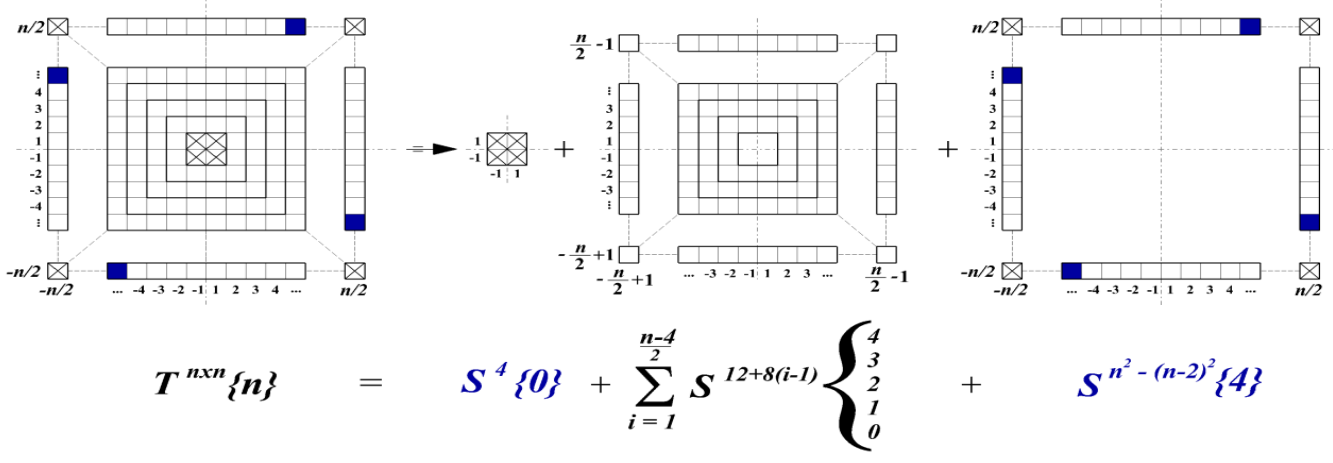

Figure 10: Case 2 when $n$ is even. Example of graphical representation. 
Case 3: The following constraints must be satisfied:

- There is one queen on subpanel $S^{4}$.

- There are three queens on subpanel $S^{n^{2}-(n-2)^{2}}$. So, at least one queen must be placed into one of the corner squares of this subpanel.

- The number of queens to be distributed on the subpanels $S^{12+8(i-1)}$ is $n-4$.

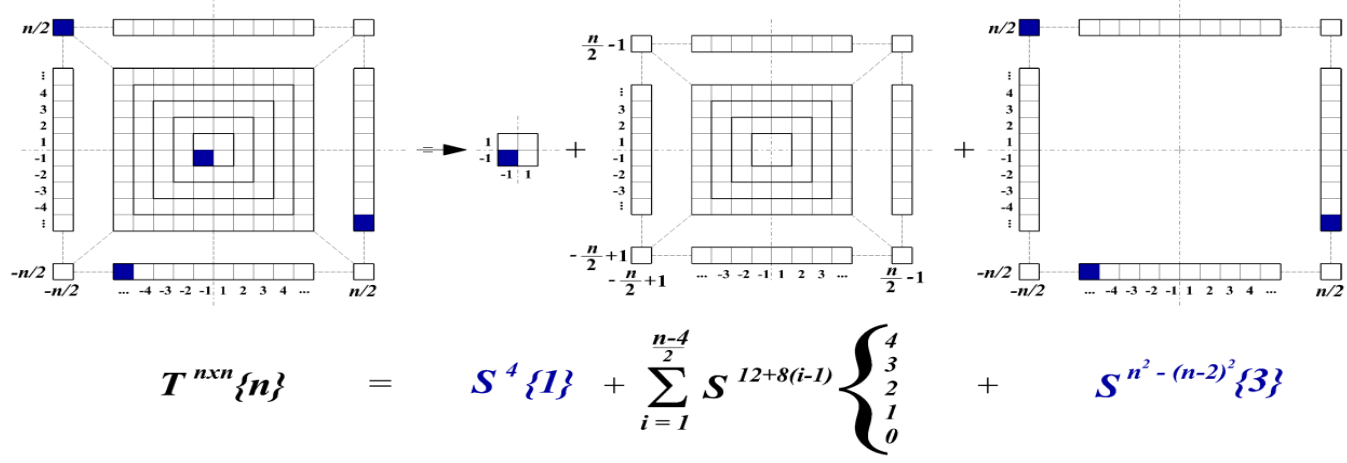

Figure 11: Case 3 when $n$ is even. Example of graphical representation

Case 4: The following constraints must be satisfied:

- There is not any queen on subpanel $S^{4}$.

- There are three queens on subpanel $S^{n^{2}-(n-2)^{2}}$. So, at least one queen must be placed into one of the corner squares of this subpanel.

- The number of queens to be distributed on the subpanels $S^{12+8(i-1)}$ is $n-3$.

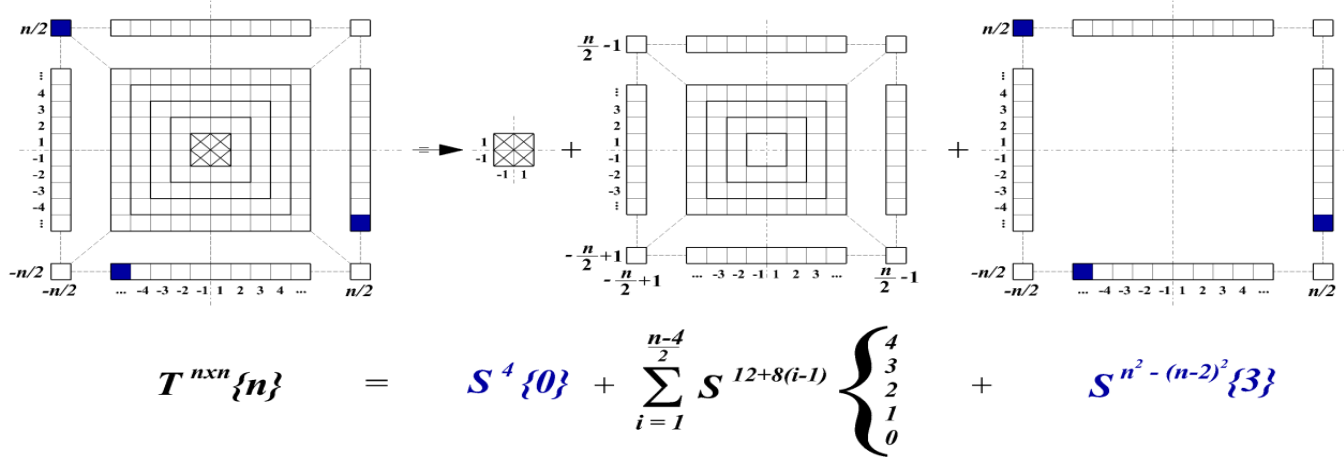

Figure 12: Case 4 when $n$ is even. Example of graphical representation.

Constraints will be configured according to the squares where some queens are already placed but in any case the following restrictions can be configured: 
- There are two alternatives on subpanel $S^{4}$; there is one queen or there is not any queen.

- There are two alternatives on subpanel $S^{n^{2}-(n-2)^{2}}$; there are four queens or there are three queens:

- If there are four queens then it is not allowed to select the corners square.

- If there are three queens then one corner square must be selected.

- It is not allowed to select more than two subpanels where a corner is selected.

- The total number of queens on the chessboard is equal to $n$.

\subsection{When $m$ is odd}

Let $m$ be an odd integer number. The number of squares on a $m \times m$ chessboard is equal to the sum of the number of squares on every subpanel:

$$
m^{2}=1+8+16+24+\cdots+m^{2}-(m-2)^{2}
$$

Let $T^{m x m}$ be a chessboard with $m \times m$ square and let $S^{1}, S^{8}, S^{16}, S^{24}, \ldots S^{m^{2}-(m-2)^{2}}$ be the subpanels. If we assume $m>3$, the several cases considering the number of queens allowed on every subpanel are defined in Equation (5).

$$
T^{m x m}\{m\}=S^{1}\left\{\begin{array}{l}
1 \\
0
\end{array}+S^{8}\left\{\begin{array}{l}
2 \\
1 \\
0
\end{array}+S^{16}\left\{\begin{array}{l}
4 \\
3 \\
2 \\
1 \\
0
\end{array}+S^{24}\left\{\begin{array}{l}
4 \\
3 \\
2 \\
1 \\
0
\end{array}+\cdots+S^{m^{2}-(m-2)^{2}}\left\{\begin{array}{l}
4 \\
3
\end{array}\right.\right.\right.\right.\right.
$$

In this case $S^{1}$ represents the first subpanel or the center of the chessboard and $S^{8}$ represents the second subpanel. Equation (5) can be expressed as the Equation (6).

$$
T^{m x m}\{m\}=S^{1}\left\{\begin{array}{l}
1 \\
0
\end{array}+S^{8}\left\{\begin{array} { l } 
{ 2 } \\
{ 1 + \sum _ { i = 1 } ^ { \frac { m - 5 } { 2 } } S ^ { 1 6 + 8 ( i - 1 ) } } \\
{ 0 }
\end{array} \left\{\begin{array} { l } 
{ 4 } \\
{ 3 } \\
{ 2 + S ^ { m ^ { 2 } - ( m - 2 ) ^ { 2 } } } \\
{ 1 } \\
{ 0 }
\end{array} \left\{\begin{array}{l}
4 \\
3
\end{array}\right.\right.\right.\right.
$$

In any case, the total number of queens in the subpanels is equal to $m$. Since the subpanels $S^{1}, S^{8}$ and $S^{m^{2}-(m-2)^{2}}$, have less choices, we will split the analysis into seven 


\begin{tabular}{|c|c|c|c|c|c|}
\hline Cases & $T^{m x m}$ & $S^{1}$ & $S^{8}$ & $\sum_{\mathrm{i}=1}^{\frac{\mathrm{m}-5}{2}} \mathrm{~S}^{16+8(\mathrm{i}-1)}$ & $\mathrm{S}^{\mathrm{m}^{2}-(\mathrm{m}-2)^{2}}$ \\
\hline Case 1 & $m$ & $\mathbf{1}$ & $\mathbf{0}$ & $m-5$ & $\mathbf{4}$ \\
\hline Case 2 & $m$ & $\mathbf{0}$ & $\mathbf{2}$ & $m-6$ & $\mathbf{4}$ \\
\hline Case 3 & $m$ & $\mathbf{0}$ & $\mathbf{1}$ & $m-5$ & $\mathbf{4}$ \\
\hline Case 4 & $m$ & $\mathbf{0}$ & $\mathbf{0}$ & $m-4$ & $\mathbf{4}$ \\
\hline Case 5 & $m$ & $\mathbf{0}$ & $\mathbf{2}$ & $m-5$ & $\mathbf{3}$ \\
\hline Case 6 & $m$ & $\mathbf{0}$ & $\mathbf{1}$ & $m-4$ & $\mathbf{3}$ \\
\hline Case 7 & $m$ & $\mathbf{0}$ & $\mathbf{0}$ & $m-3$ & $\mathbf{3}$ \\
\hline
\end{tabular}

Table 2: List of seven possible cases when $m$ is odd.

cases depending on the number of queens in these subpanels. They are defined in the Table 2

Depending on the number of queens allowed on the subpanels $\mathrm{S}^{16+8(\mathrm{i}-1)}$, there are several possible subcases. It is represented in Equation (7).

$$
\sum_{i=1}^{\frac{m-5}{2}} S^{16+8(i-1)}=\left\{\begin{array}{l}
\text { Case } 1: m-5 \\
\text { Case } 2: m-6 \\
\text { Case } 3: m-5 \\
\text { Case } 4: m-4 \\
\text { Case } 5: m-5 \\
\text { Case } 6: m-4 \\
\text { Case } 7: m-3
\end{array}\right.
$$

Now we proceed to analyze each case one by one.

Case 1: The following constraints must be satisfied:

- There is one queen on subpanel $S^{1}$. So, it is not allowed to place any queen in the principal diagonals and central squares of the board.

- There is not any queen on subpanel $S^{8}$.

- There are four queens on subpanel $S^{m^{2}-(m-2)^{2}}$.

- The number of queens to be distributed on the subpanels $S^{16+8(i-1)}$ is $m-5$. 


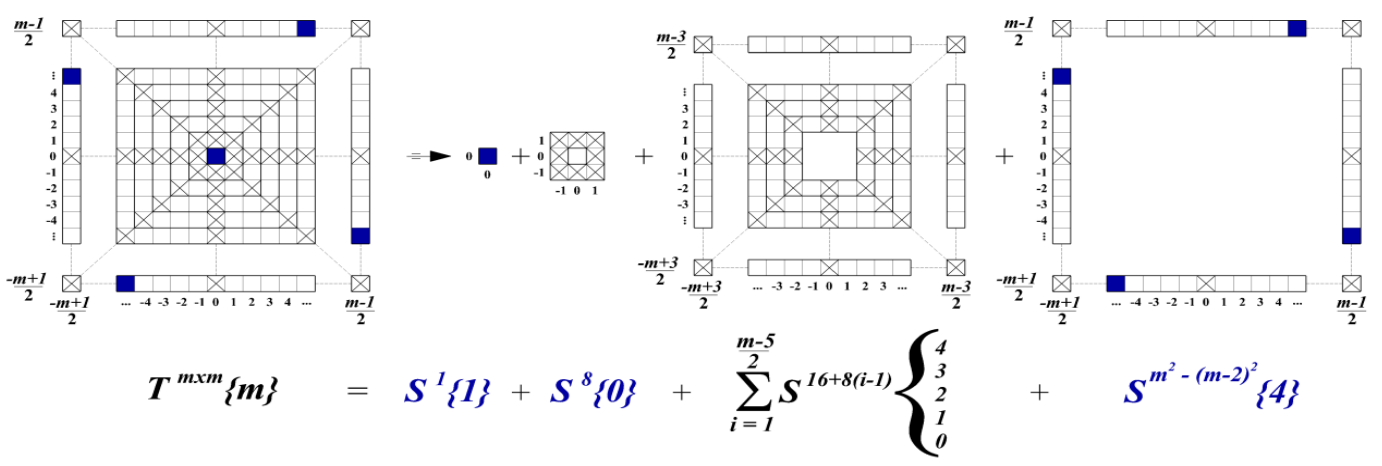

Figure 13: Case 1 when $m$ is odd. Example of graphical representation.

Case 2: The following constraints must be satisfied:

- There is not any queen on subpanel $S^{1}$.

- There are two queens on subpanel $S^{8}$. So, at least one queen must be placed into one of the corner squares of this subpanel.

- There are four queens on subpanel $S^{m^{2}-(m-2)^{2}}$. So, it is not allowed to place any queen in the corner squares of this subpanel.

- The number of queens to be distributed on the subpanels $S^{16+8(i-1)}$ is $m-6$.

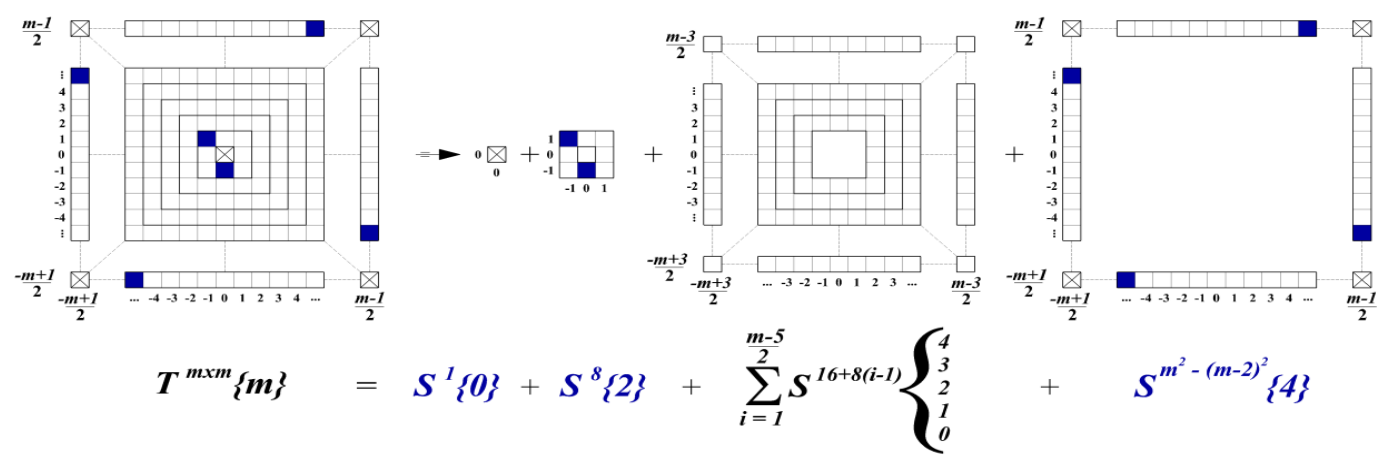

Figure 14: Case 2 when $m$ is odd. Example of graphical representation.

Case 3: The following constraints must be satisfied:

- There is not any queen on subpanel $S^{1}$. 
- There is one queen on subpanel $S^{8}$.

- There are four queens on subpanel $S^{m^{2}-(m-2)^{2}}$. So, it is not allowed to place any queen in the corner squares of this subpanel.

- The number of queens to be distributed on the subpanels $S^{16+8(i-1)}$ is $m-5$.

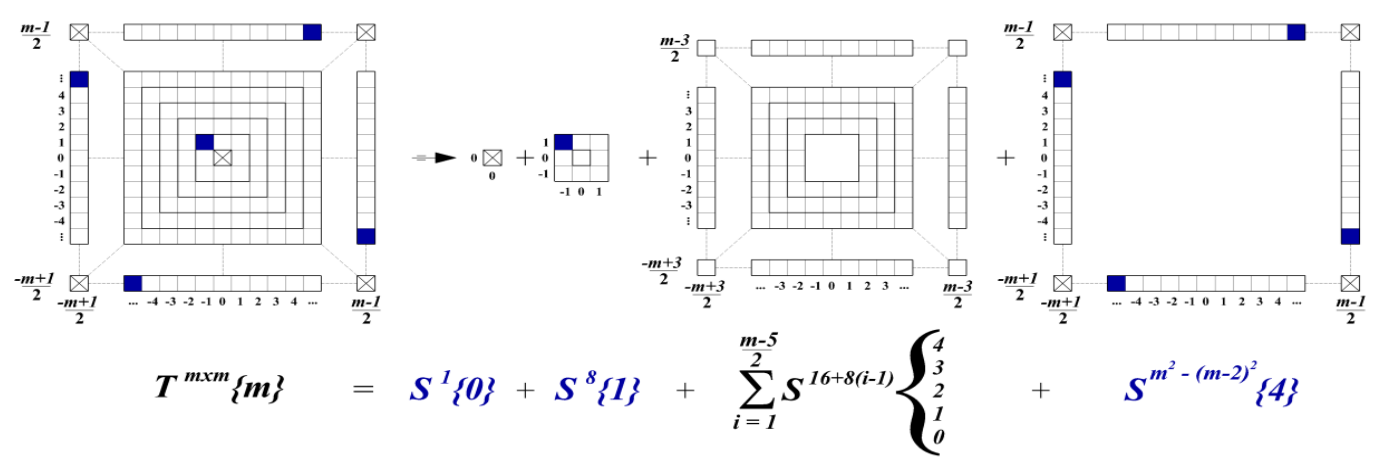

Figure 15: Case 3 when $m$ is odd. Example of graphical representation.

Case 4: The following constraints must be satisfied:

- There is not any queen on subpanel $S^{1}$.

- There is not any queen on subpanel $S^{8}$.

- There are four queens on subpanel $S^{m^{2}-(m-2)^{2}}$. So, it is not allowed to place any queen in the corner squares of this subpanel.

- The number of queens to be distributed on the subpanels $S^{16+8(i-1)}$ is $m-4$.

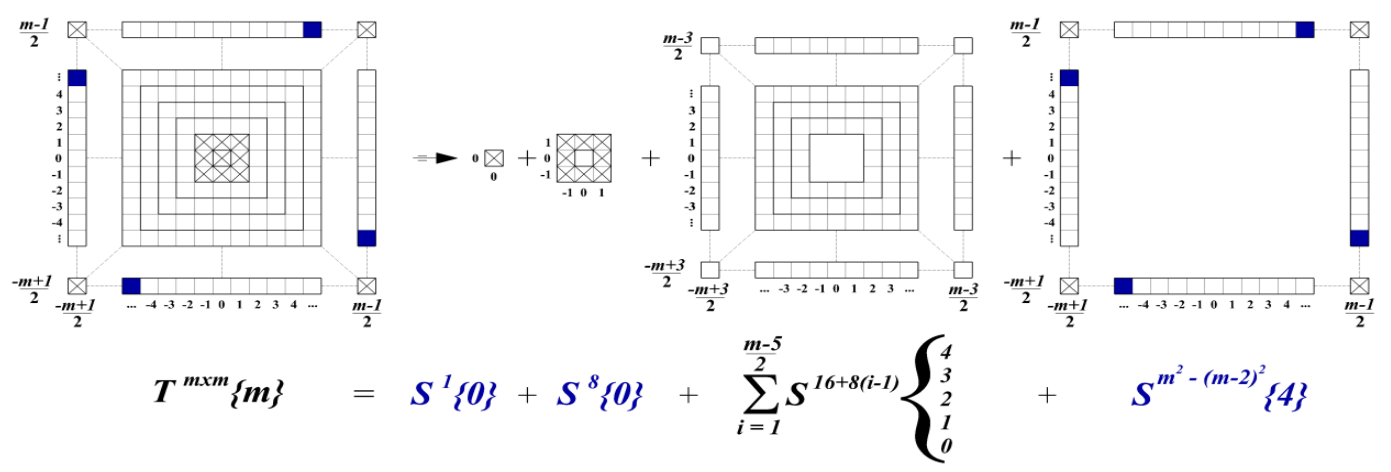

Figure 16: Case 4 when $m$ is odd. Example of graphical representation 
Case 5: The following constraints must be satisfied:

- There is not any queen on subpanel $S^{1}$.

- There are two queens on subpanel $S^{8}$.

- There are three queens on subpanel $S^{m^{2}-(m-2)^{2}}$. So, at least one queen must be placed into one of the corner squares of this subpanel.

- The number of queens to be distributed on the subpanels $S^{16+8(i-1)}$ is $m-5$.

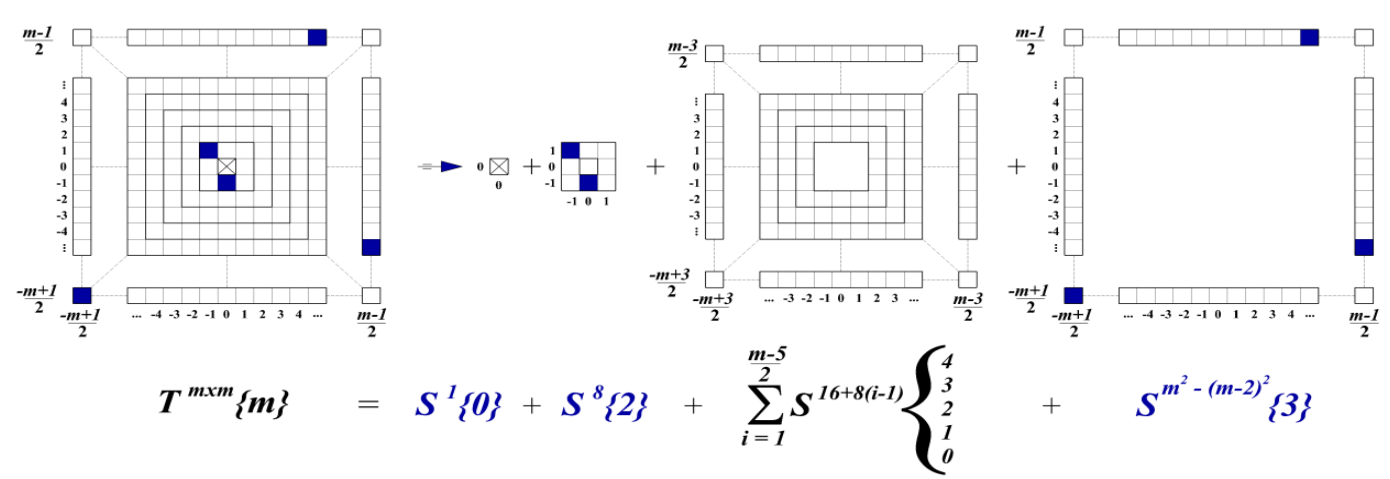

Figure 17: Case 5 when $m$ is odd. Example of graphical representation.

Case 6: The following constraints must be satisfied:

- There is not any queen on subpanel $S^{1}$.

- There is one queen on subpanel $S^{8}$.

- There are three queens on subpanel $S^{m^{2}-(m-2)^{2}}$. So, at least one queen must be placed into one of the corner squares of this subpanel.

- The number of queens to be distributed on the subpanels $S^{16+8(i-1)}$ is $m-4$. 


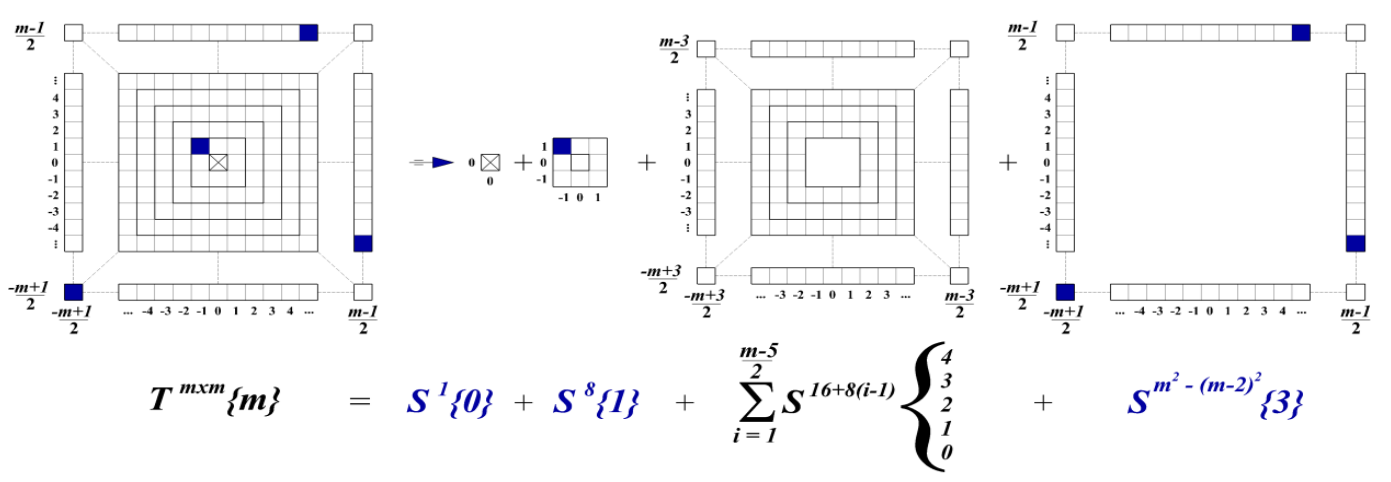

Figure 18: Case 6 when $m$ is odd. Example of graphical representation.

Case 7: The following constraints must be satisfied:

- There is not any queen on subpanel $S^{1}$.

- There is not any queen on subpanel $S^{8}$.

- There are three queens on subpanel $S^{m^{2}-(m-2)^{2}}$. So, at least one queen must be placed into one of the corner squares of this subpanel.

- The number of queens to be distributed on the subpanels $S^{16+8(i-1)}$ is $m-3$.

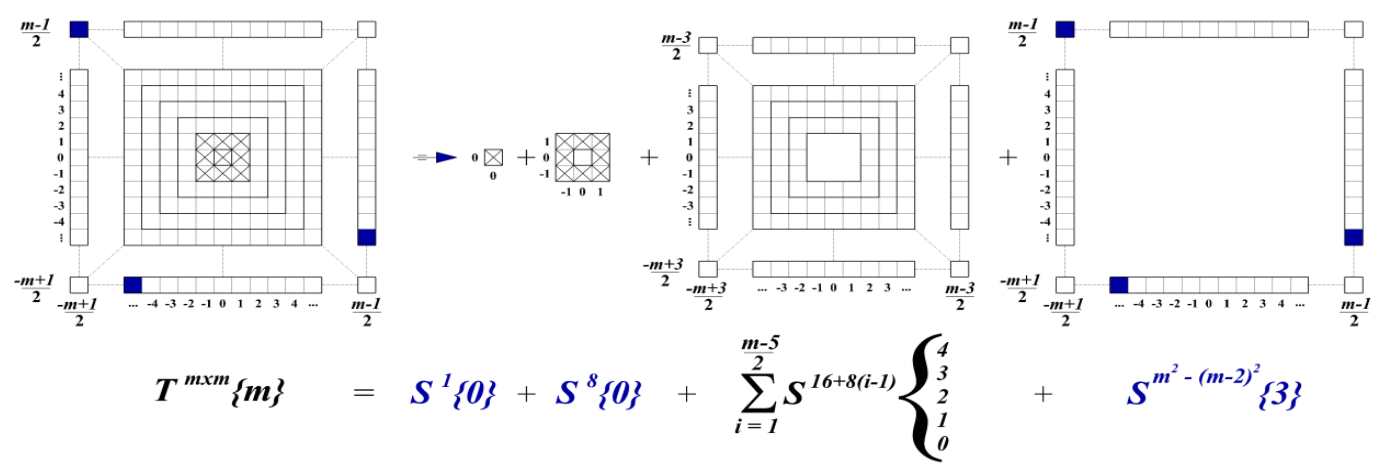

Figure 19: Case 7 when $m$ is odd. Example of graphical representation.

Constraints will be configured according to the squares where some queens are already placed but in any case the following restrictions can be configured:

- There are two alternatives on subpanel $S^{1}$; there is one queen or there is not any queen. If there is one queen then it is not allowed to place any queen in the principal diagonals of the board. 
- There are three alternatives on subpanel $S^{8}$; there are two queens, there is one queen or there is not any queen. If there are two queens on this subpanel then one corner square must be selected.

- There are two alternatives on subpanel $S^{m^{2}-(m-2)^{2}}$; there are four queens or there are three queens:

- If there are four queens then it is not allowed to select the corners square.

- If there are three queens then one corner square must be selected.

- It is not allowed to select more than two subpanels where a corner is selected.

- The total number of queens on the chessboard is equal to $m$

Finally, the cases and sub-cases list will depend on:

- The number of queens pre-placed; in general, when the number of queens pre-placed increase, the list of sub-cases decrease.

- The position of the queens preplaced; for example, if $n$ is even and there is one queen on subpanel $S^{4}$ and three or four queens on subpanel $S^{n^{2}-(n-2)^{2}}$ then the list of sub-cases would decrease.

A complete analysis of a completion problem in a $8 \times 8$ chessboard is included in the Appendix.

\subsection{Pattern rules for extended solutions}

This section shows how the method for breaking down an $n$ by $n$ chessboard could be apply for finding repetitive patterns of solutions.

Let $C>0$ be an even integer number. A solution of the $n$-Queens puzzle can be extended to another solution of the $n^{\prime}$-Queens puzzle with $n^{\prime}=n+C$. The value of $C$ is a proportional relation between the number of subpanels of $n$ and $n^{\prime}$. The pattern rule we propose is based on the sequence of the number of queens placed from the lowest to the highest subpanels of the smallest chessboard and also on a repetitiveness in the position of the queens on the chessboard for dimension $n$ to dimension $n^{\prime}$.

Now two example solutions for dimension $n=8$ will be extended for higher dimension.

Example 1. In Figure 20 we show a solution of the 8-Queens puzzle where several colors have been used for helping to the searching of the pattern.

According to the number of queens placed on subpanels and the position of the queens on the chessboard, some hypothesis can be assumed: 


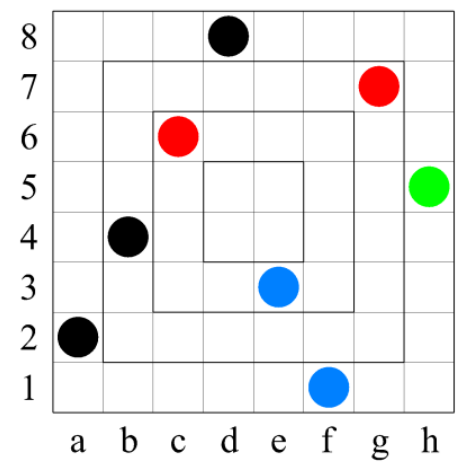

Figure 20: Example of 8-Queen puzzle solution.

- There won't be any queen on the first subpanel $S^{4}$ and there will be four queens on the last subpanel $S^{n^{2}-(n-2)^{2}}$

- Two queens will be placed on the corners of the internal subpanels $S^{12+8(i-1)}$ (marked in red color).

- The positions of the queens will keep some repetitiveness for dimension $n$ to dimension $n^{\prime}$

The value of $n^{\prime}$ can be found by testing $C>0$. Figure 21 shows a solution for $n^{\prime}=20$ as an extended solution of $n=8$.

The number of queens placed on every subpanel with $C=12$ and for $n=8$ to extended solutions is showed in Table 3 ,

\begin{tabular}{|c|c|c|c|c|c|c|c|c|c|c|c|c|c|c|c|c|c|}
\hline \multicolumn{10}{|c|}{ Subpanels } \\
\hline$n$ & $\mathbf{4}$ & $\mathbf{1 2}$ & $\mathbf{2 0}$ & $\mathbf{2 8}$ & $\mathbf{3 6}$ & $\mathbf{4 4}$ & $\mathbf{5 2}$ & $\mathbf{6 0}$ & $\mathbf{6 8}$ & $\mathbf{7 6}$ & $\mathbf{8 4}$ & $\mathbf{9 2}$ & $\mathbf{1 0 0}$ & $\mathbf{1 0 8}$ & $\mathbf{1 1 6}$ & $\mathbf{1 2 4}$ & $\ldots$ \\
\hline 8 & 0 & 2 & 2 & 4 & & & & & & & & & & & & & \\
\hline 20 & 0 & 0 & 0 & 2 & 2 & 4 & 2 & 4 & 2 & 4 & & & & & & & \\
\hline 32 & 0 & 0 & 0 & 0 & 0 & 2 & 2 & 4 & 2 & 4 & 2 & 4 & 2 & 4 & 2 & 4 & \\
\hline 44 & 0 & 0 & 0 & 0 & 0 & 0 & 0 & 2 & 2 & 4 & 2 & 4 & 2 & 4 & 2 & 4 & $\ldots$ \\
\hline$\ldots$ & 0 & 0 & 0 & 0 & 0 & 0 & 0 & 0 & 0 & 2 & $\ldots$ & & & & & & \\
\hline
\end{tabular}

Table 3: Representation of the number of queens on subpanels for Example 1.

One approach is:

- There is not any queen on subpanel $S^{4}$.

- There are four queens on subpanel $S^{n^{2}-(n-2)^{2}}$. 


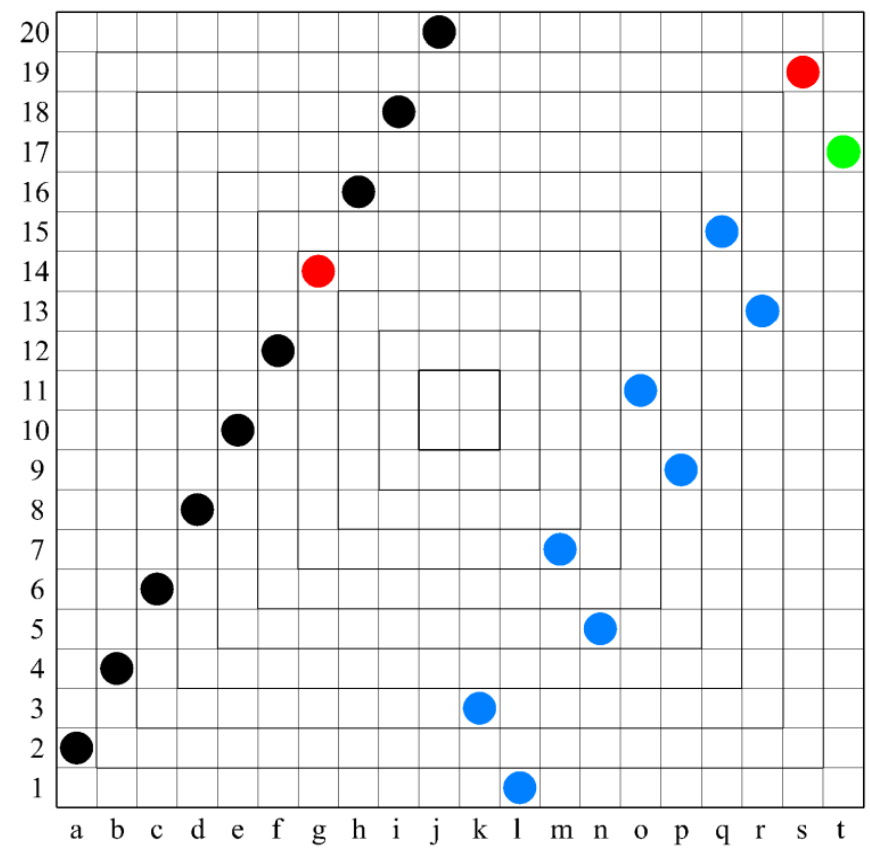

Figure 21: Example of 20-Queens puzzle solution.

- The number of subpanels with zero queens for dimension $n=8$ to higher dimensions will follow the sequence $1-3-5-7 \ldots$

- A subpanel with two queens will be fixed after the last subpanel with zero queens and one queen must be placed on the top left corner square (red color).

- The number of subpanels with two queens for dimension $n=8$ to higher dimensions will follow the sequence $2-4-6-8 \ldots$

- The number of subpanels with four queens for dimension $n=8$ to higher dimensions will follow the sequence $1-3-5-7 \ldots$

The number of queens to be put on the subpanels is showed in the compact representation.

$$
T^{n \times n}\{n\}=\sum_{i=0}^{\frac{n-8}{6}} S^{4+8 i}\{0\}+S^{\frac{4}{3}(n+1)}\{2\}+\sum_{i=\frac{n+4}{12}}^{\frac{n-4}{4}} S^{4+16 i}\{2\}+\sum_{i=\frac{n+4}{12}}^{\frac{n-4}{4}} S^{12+16 i}\{4\}
$$

For instance, the equation to determine the number of queens to put on the subpanels for $n^{\prime}=2000$ is showed in the following compact representation. 


$$
T^{4000000}\{2000\}=\sum_{i=0}^{332} S^{4+8 i}\{0\}+S^{2668}\{2\}+\sum_{i=167}^{499} S^{4+16 i}\{2\}+\sum_{i=167}^{499} S^{12+16 i}\{4\}
$$

Example 2. In Figure 22 we show another solution of the 8-Queens puzzle where several colors have been used for helping to the searching of the pattern.

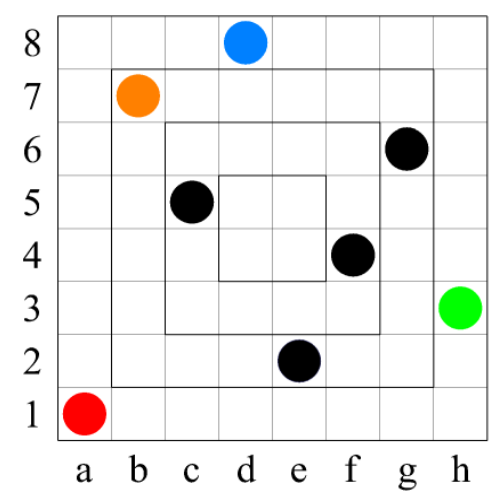

Figure 22: Example of 8-Queens puzzle solution.

According to the number of queens placed on subpanels and the position of the queens on the chessboard, some hypothesis can be assumed:

- There won't be any queen on the first subpanel $S^{4}$ and there will be three queens on the last subpanel $S^{n^{2}-(n-2)^{2}}$.

- One queen will be placed on the corner of the internal subpanels $S^{12+8(i-1)}$ (marked in orange color).

- The positions of the queens will keep some repetitiveness for dimension $n$ to dimension $n^{\prime}$.

The value of $n^{\prime}$ can be found by testing $C>0$. Figure 23 shows a solution for $n^{\prime}=20$ as an extended solution of $n=8$.

The number of queens placed on every subpanel with $C=12$ and for $n=8$ to extended solutions is showed in Table 4 ,

One approach is:

- There is not any queen on subpanel $S^{4}$.

- There are three queens on subpanel $S^{n^{2}-(n-2)^{2}}$. 


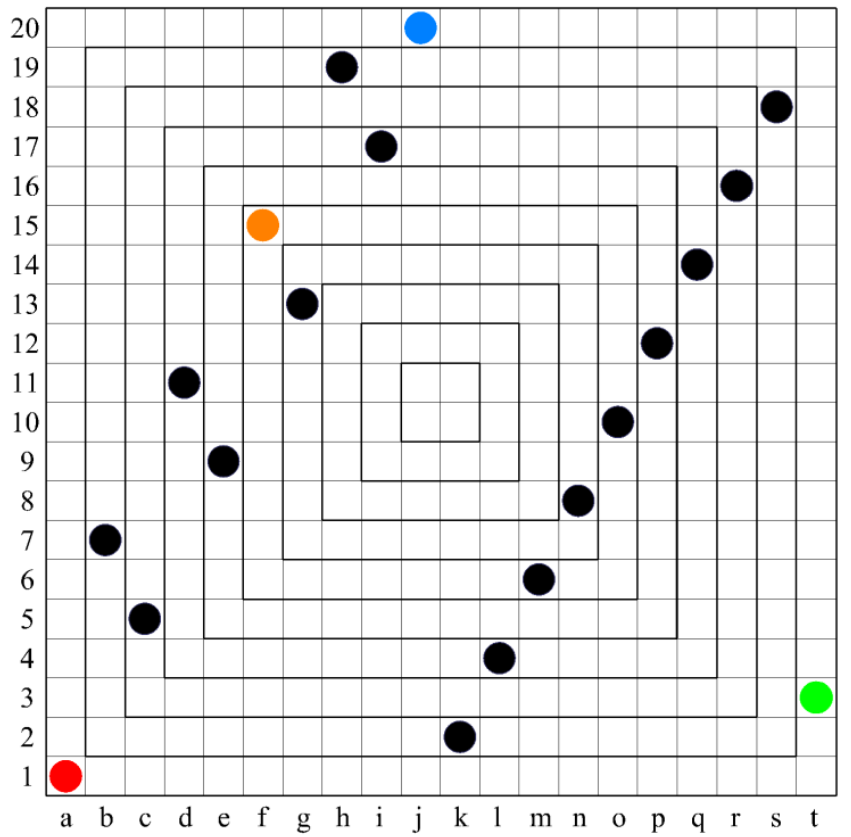

Figure 23: Example of 20-Queen puzzle solution.

\begin{tabular}{|c|c|c|c|c|c|c|c|c|c|c|c|c|c|c|c|c|c|}
\hline & \multicolumn{110}{|c|}{ Subpanels } \\
\hline$n$ & $\mathbf{4}$ & $\mathbf{1 2}$ & $\mathbf{2 0}$ & $\mathbf{2 8}$ & $\mathbf{3 6}$ & $\mathbf{4 4}$ & $\mathbf{5 2}$ & $\mathbf{6 0}$ & $\mathbf{6 8}$ & $\mathbf{7 6}$ & $\mathbf{8 4}$ & $\mathbf{9 2}$ & $\mathbf{1 0 0}$ & $\mathbf{1 0 8}$ & $\mathbf{1 1 6}$ & $\mathbf{1 2 4}$ & $\ldots$ \\
\hline 8 & 0 & 2 & 3 & 3 & & & & & & & & & & & & & \\
\hline 20 & 0 & 0 & 0 & 2 & 3 & 2 & 4 & 2 & 4 & 3 & & & & & & & \\
\hline 32 & 0 & 0 & 0 & 0 & 0 & 2 & 3 & 2 & 4 & 2 & 4 & 2 & 4 & 2 & 4 & 3 & \\
\hline 44 & 0 & 0 & 0 & 0 & 0 & 0 & 0 & 2 & 3 & 2 & 4 & 2 & 4 & 2 & 4 & 2 & $\ldots$ \\
\hline$\ldots$ & 0 & 0 & 0 & 0 & 0 & 0 & 0 & 0 & 0 & 2 & 3 & 2 & 4 & $\ldots$ & & & \\
\hline
\end{tabular}

Table 4: Representation of the number of queens on subpanels for Example 2.

- The number of subpanels with zero queens for dimension $n=8$ to higher dimensions will follow the sequence $1-3-5-7 \ldots$

- A subpanel with two queens will be fixed after the last subpanel with zero queens.

- A subpanel with three queens will be fixed after the first subpanel with two queens and one queen must be placed on the top left corner square (orange color).

- The number of subpanels with two queens for dimension $n=8$ to higher dimensions will follow the sequence $1-3-5-7 \ldots$ 
- The number of subpanels with four queens for dimension $n=8$ to higher dimensions will follow the sequence $0-2-4-6 \ldots$

The number of queens to be put on the subpanels is showed in the compact representation.

$$
\begin{aligned}
T^{n \times n}\{n\}=\sum_{i=0}^{\frac{n-8}{6}} S^{4+8 i}\{0\}+S^{\frac{4}{3}(n+1)}\{2\} & +S^{\frac{4}{3}(n+7)}\{3\}+\sum_{i=\frac{n+4}{12}}^{\frac{n-8}{4}} S^{12+16 i}\{2\}+ \\
& +\sum_{i=\frac{n+4}{12}}^{\frac{n-8}{4}} S^{20+16 i}\{4\}+S^{28+4(n-8)}\{3\}
\end{aligned}
$$

In general, solutions of the $n$-Queens puzzle can be extended to higher dimensions and it could be applied for solving $n$-Queens completion problems. 


\section{Appendix: Complete analysis of a completion problem in a $8 \times 8$ chessboard}

In this appendix we will give a complete example of how the algorithm presented in this paper would be applied to solve the 8-Queens completion puzzle, in the instance proposed by Nauck in 1850 [ACJ18, see Figure 1. Two queens are already placed on the chessboard and the solver is asked for placing the other six queens.

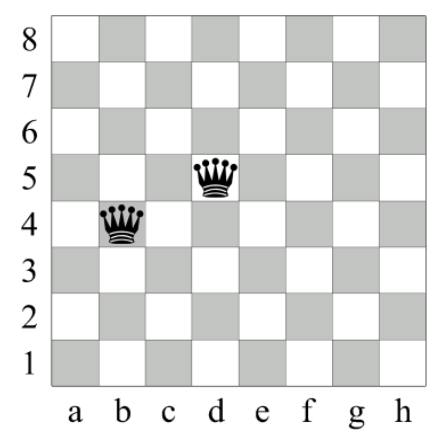

Figure 1: Example of two queens preplaced on the chessboard.

According to our algorithm we have the following constraint on the subpanels:

- There is one queen placed on subpanel $S^{4}$ and it must be placed on d5 square.

- There is at least one queen on subpanel $S^{20}$ and it must be placed on b4 square.

- The total number of queens in the subpanels $S^{4}, S^{12}, S^{20}$ and $S^{28}$ must be equal to eight.

We can specify Equation (1) putting only the cases allowed by the position of the queens which are already on the board:

$$
\mathrm{T}^{64}\{8\}=\mathrm{S}^{4}\{1\}+\mathrm{S}^{12}\left\{\begin{array}{l}
4 \\
3 \\
2 \\
1 \\
0
\end{array}+\mathrm{S}^{20}\left\{\begin{array}{l}
4 \\
3 \\
2 \\
1
\end{array}+\mathrm{S}^{28}\left\{\begin{array}{l}
4 \\
3
\end{array}\right.\right.\right.
$$

The fact that the subpanel $S^{4}$ has only one possibility reduces the cases. It is represented in Table 1. 


\begin{tabular}{|l|c|c|c|c|c|}
\hline & $\mathrm{T}^{8 x 8}$ & $\mathrm{~S}^{4}$ & $\mathrm{~S}^{12}$ & $\mathrm{~S}^{20}$ & $\mathrm{~S}^{28}$ \\
\hline Case 1 & 8 & 1 & - & - & 4 \\
\hline Case 2 & 8 & 1 & - & - & 3 \\
\hline
\end{tabular}

Table 1: List of two possible cases for the example.

Case 1: There is one queen on subpanel $S^{4}$, there are four queens on subpanel $S^{28}$ and there is at least one queen on subpanel $S^{20}$. It is represented in Equation (2).

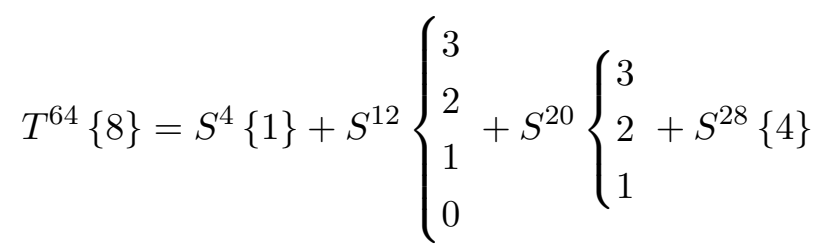

In this case, the number of queens to be distributed on subpanels $S^{12}$ and $S^{20}$ must be equal to 3. It is represented in Equation (3).

$$
S^{12}\left\{\begin{array} { l } 
{ 2 } \\
{ 1 + S ^ { 2 0 } } \\
{ 0 }
\end{array} \left\{\begin{array}{l}
3 \\
2 \\
1
\end{array}=3\right.\right.
$$

Case 2: There is one queen on subpanel $S^{4}$, there are three queens on subpanel $S^{28}$ and there is at least one queen on subpanel $S^{20}$. It is represented in Equation (4).

$$
T^{64}\{8\}=S^{4}\{1\}+S^{12}\left\{\begin{array}{l}
3 \\
2 \\
1 \\
0
\end{array}+S^{20}\left\{\begin{array}{l}
4 \\
3 \\
2 \\
1
\end{array} \quad+S^{28}\{3\}\right.\right.
$$

In this case, the number of queens to be distributed on subpanels $S^{12}$ and $S^{20}$ must be equal to 4. It is represented in Equation (5).

$$
S^{12}\left\{\begin{array}{l}
3 \\
2 \\
1 \\
0
\end{array}+S^{20}\left\{\begin{array}{l}
4 \\
3 \\
2 \\
1
\end{array}=4\right.\right.
$$

Subcases allowed are listed in Table 2 and represented in Figure 2 .

Now we proceed to analyze each subcase one by one. The solutions will be found depending on subcases constraints. 


\begin{tabular}{|c|c|c|c|c|c|}
\hline Sub-cases & $\mathbf{T}^{64}$ & $\mathbf{S}^{4}$ & $\mathbf{S}^{12}$ & $\mathbf{S}^{20}$ & $\mathbf{S}^{28}$ \\
\hline Sub-case 1.1 & 8 & 1 & $\mathbf{0}$ & $\mathbf{3}$ & 4 \\
\hline Sub-case 1.2 & 8 & 1 & $\mathbf{1}$ & $\mathbf{2}$ & 4 \\
\hline Sub-case 1.3 & 8 & 1 & $\mathbf{2}$ & $\mathbf{1}$ & 4 \\
\hline Sub-case 2.1 & 8 & 1 & $\mathbf{0}$ & $\mathbf{4}$ & 3 \\
\hline Sub-case 2.2 & 8 & 1 & $\mathbf{1}$ & $\mathbf{3}$ & 3 \\
\hline Sub-case 2.3 & 8 & 1 & $\mathbf{2}$ & $\mathbf{2}$ & 3 \\
\hline Sub-case 2.4 & 8 & 1 & $\mathbf{3}$ & $\mathbf{1}$ & 3 \\
\hline
\end{tabular}

Table 2: List of sub-cases allowed for cases 1 and 2 .

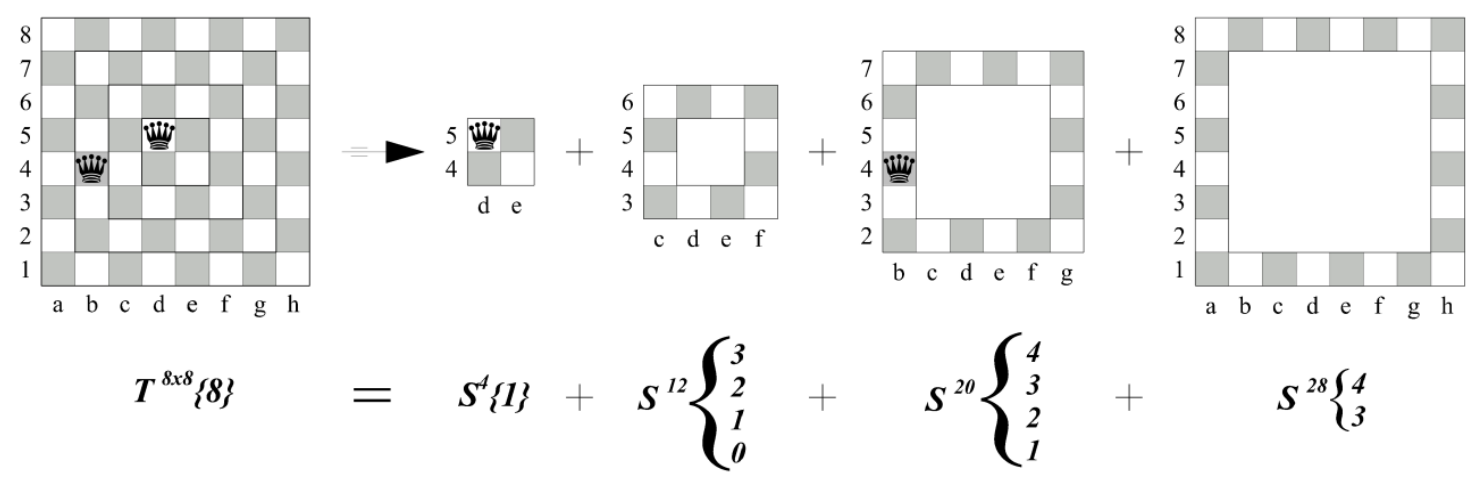

Figure 2: Graphical representation of subcases allowed.

Sub-case 1.1: There is no queen on subpanel $S^{12}$ and there are three queens on subpanel $S^{20}$. (See Table 2)

The following constraints can be configured:

- There is one queen on subpanel $S^{4}$ and it must be placed in $\mathrm{d} 5$ square.

- There is not any queen on subpanel $S^{12}$.

- There are three queens on subpanel $S^{20}$ and one must be placed in b4 square.

- There are four queens on subpanel $S^{28}$. So, it is not allowed to place any queen in a1, a8, h1 and h8 squares.

Chessboard reconstruction from subpanel $S^{4}$ is represented in Figure 3 . The squares allowed for placing a queen on subpanel $S^{20}$ are c2, e2, f2, g3, g6, g7 and c7.

To satisfy the constraints for placing three queens on subpanel $S^{20}$, the alternatives are $\{b 4, \mathrm{c} 2, \mathrm{~g} 3\},\{\mathrm{b} 4, \mathrm{c} 2, \mathrm{~g} 7\},\{\mathrm{b} 4, \mathrm{e} 2, \mathrm{~g} 3\},\{\mathrm{b} 4, \mathrm{e} 2, \mathrm{~g} 6\},\{\mathrm{b} 4, \mathrm{e} 2, \mathrm{~g} 7\},\{\mathrm{b} 4, \mathrm{e} 2, \mathrm{c} 7\},\{\mathrm{b} 4$, 


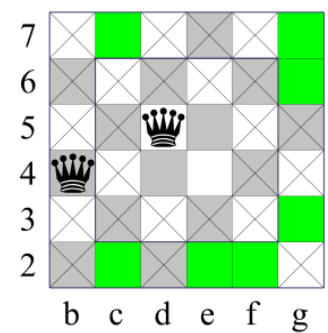

Figure 3: Sub-case 1.1. Squares allowed on sub-panel $S^{20}$.
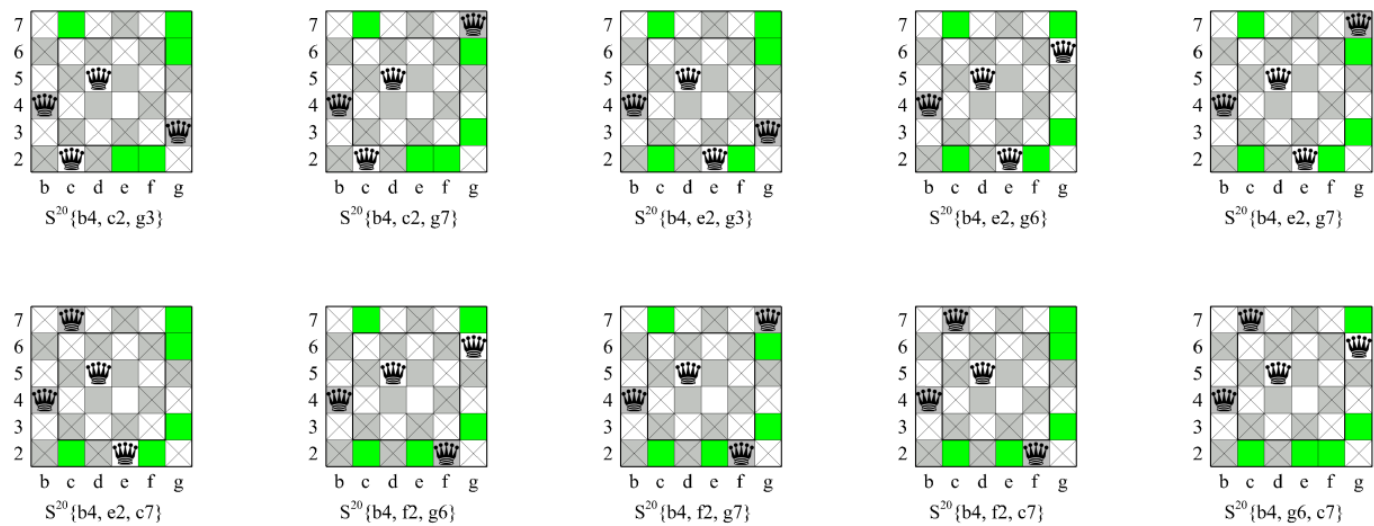

Figure 4: Sub-case 1.1. Graphical representation of the several alternatives for placing three queens on subpanel $S^{20}$.

$\mathrm{f} 2, \mathrm{~g} 6\},\{\mathrm{b} 4, \mathrm{f} 2, \mathrm{~g} 7\},\{\mathrm{b} 4, \mathrm{f} 2, \mathrm{c} 7\}$ and $\{\mathrm{b} 4, \mathrm{~g} 6, \mathrm{c} 7\}$ squares. Figures 4 and 5 represent the several alternatives.

- If $\{\mathrm{b} 4, \mathrm{c} 2, \mathrm{~g} 3\}$ squares are selected then the squares allowed on subpanel $S^{28}$ are a7, $\mathrm{a} 6, \mathrm{f} 1, \mathrm{~h} 6$ and $\mathrm{e} 8$. The squares a1 and h8 are not allowed because of the constraint to put four queens on subpanel $S^{28}$, and not for the constraints of the queens selected on subpanel $S^{20}$. To satisfy the constraints for placing four queens on subpanel $\mathrm{S}^{28}$, the alternatives are $\{\mathrm{a} 6, \mathrm{f} 1, \mathrm{~h} 6, \mathrm{e} 8\}$ and $\{\mathrm{a} 7, \mathrm{f} 1, \mathrm{~h} 6, \mathrm{e} 8\}$. If $\{\mathrm{a} 6, \mathrm{f} 1$, $\mathrm{h} 6, \mathrm{e} 8\}$ is selected no solution is possible because a queen placed in a6 square would be attacking to a queen placed in $\mathrm{f} 1$ or $\mathrm{h} 6$ squares. If $\{\mathrm{a} 7, \mathrm{f} 1, \mathrm{~h} 6, \mathrm{e} 8\}$ is selected there is a solution because none of the queens is attacking another queen.

- If $\{\mathrm{b} 4, \mathrm{c} 2, \mathrm{~g} 7\}$ squares are selected then the squares allowed on subpanel $S^{28}$ are $\mathrm{a} 6, \mathrm{f} 1, \mathrm{~h} 3$ and $\mathrm{e} 8$. No solution is possible because a queen placed in $\mathrm{f} 1$ square would be attacking to a queen placed in a6 or h3 square. 
- If $\{\mathrm{b} 4, \mathrm{e} 2, \mathrm{~g} 3\}$ squares are selected then the squares allowed on subpanel $S^{28}$ are a7, c1, h6, h7 and c8. No solution is possible because a queen placed in c1 square would be attacking to a queen placed in c8 square.

- If $\{\mathrm{b} 4, \mathrm{e} 2, \mathrm{~g} 6\}$ squares are selected then the squares allowed on subpanel $S^{28}$ are a7, c1, h3 and c8. No solution is possible because a queen placed in c1 square would be attacking to a queen placed in c8 square.

- If $\{\mathrm{b} 4, \mathrm{e} 2, \mathrm{~g} 7\}$ squares are selected then the squares allowed on subpanel $S^{28}$ are $\mathrm{c} 1$, h3 and c8. No solution is possible because it is not allowed to put four queens on subpanel $S^{28}$.

- If $\{\mathrm{b} 4, \mathrm{e} 2, \mathrm{c} 7\}$ squares are selected then the squares allowed on subpanel $S^{28}$ are $\mathrm{g} 1, \mathrm{~h} 3$ and h6. No solution is possible because it is not allowed to put four queens on subpanel $S^{28}$.

- If $\{\mathrm{b} 4, \mathrm{f} 2, \mathrm{~g} 6\}$ squares are selected then the squares allowed on subpanel $S^{28}$ are $\mathrm{c} 1, \mathrm{~h} 3$ and $\mathrm{c} 8$. No solution is possible because it is not allowed to put four queens on subpanel $S^{28}$.

- If $\{\mathrm{b} 4, \mathrm{f} 2, \mathrm{~g} 7\}$ squares are selected then the squares allowed on subpanel $S^{28}$ are a6, c1, h3, e8 and c8. To satisfy the constraints for placing four queens on subpanel $S^{28}$, the alternatives are $\{\mathrm{a} 6, \mathrm{c} 1, \mathrm{~h} 3, \mathrm{c} 8\},\{\mathrm{a} 6, \mathrm{c} 1, \mathrm{~h} 3, \mathrm{e} 8\}$ and $\{\mathrm{a} 6, \mathrm{~h} 3, \mathrm{e} 8, \mathrm{c} 8\}$. If $\{a 6, c 1, h 3, c 8\}$ is selected there is not solution because a queen placed in $\mathrm{c} 8$ square would be attacking to a queen placed in a6 or c1 squares. If $\{a 6, \mathrm{c} 1, \mathrm{~h} 3$, $\mathrm{e} 8\}$ is selected there is a solution because none of the queens is attacking another queen. If $\{\mathrm{a} 6, \mathrm{~h} 3, \mathrm{e} 8, \mathrm{c} 8\}$ is selected no solution is possible because a queen placed in e8 square would be attacking to a queen placed in $\mathrm{c} 8$.

- If $\{\mathrm{b} 4, \mathrm{f} 2, \mathrm{c} 7\}$ squares are selected then the squares allowed on subpanel $S^{28}$ are a6, $\mathrm{h} 3, \mathrm{~h} 6$ and e8. No solution is possible because it is not allowed to put four queens on subpanel $S^{28}$.

- If $\{\mathrm{b} 4, \mathrm{~g} 6, \mathrm{c} 7\}$ squares are selected then the squares allowed on subpanel $S^{28}$ are f1 and h3. No solution is possible because it is not allowed to put four queens on subpanel $S^{28}$.

Sub-case 1.2: There is one queen on subpanel $S^{12}$ and there are two queens on subpanel $S^{20}$. (See Table 2)

The following constraints can be configured:

- There is one queen on subpanel $S^{4}$ and it must be placed in d5 square.

- There is one queen on subpanel $S^{12}$. 

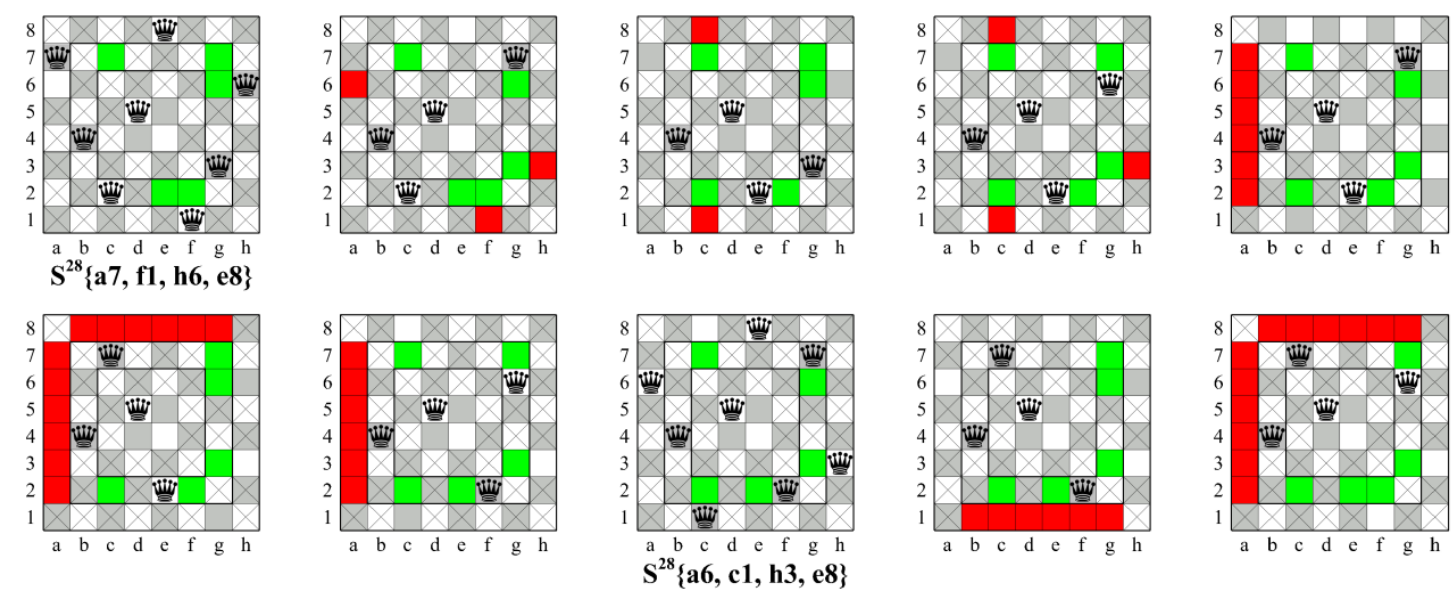

Figure 5: Subcase 1.1. Graphical representation of the several alternatives for placing four queens on subpanel $S^{28}$.

- There are two queens on subpanel $S^{20}$ and one must be placed in b4 square.

- There are four queens on subpanel $S^{28}$. So, it is not allowed to place any queen in a1, a8, h1 and h8 squares.

Chessboard reconstruction from subpanel $S^{4}$ is represented in Figure 6. The squares allowed on subpanel $S^{12}$ are e3 and f6, and the squares allowed on subpanel $S^{20}$ are c2, e2, f2, g3, g6, g7 and c7.

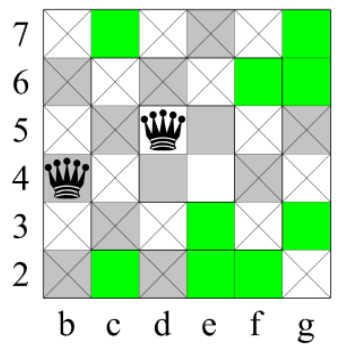

Figure 6: Sub-case 1.2. Squares allowed on sub-panels $\mathrm{S}^{12}$ y $\mathrm{S}^{20}$.

To satisfy the constraints for placing one queen on subpanel $S^{12}$, the alternatives are e3 or f6 squares. Figure 7 represents the two alternatives.

- If e3 square is selected on subpanel $S^{12}$, then the squares allowed on subpanel $S^{20}$ are c2, g6, g7 and c7. To satisfy the constraint for placing two queens on subpanel $\mathrm{S}^{20}$, the alternatives are $\{\mathrm{b} 4, \mathrm{c} 2\},\{\mathrm{b} 4, \mathrm{~g} 6\},\{\mathrm{b} 4, \mathrm{~g} 7\}$ or $\{\mathrm{b} 4, \mathrm{c} 7\}$ squares. 

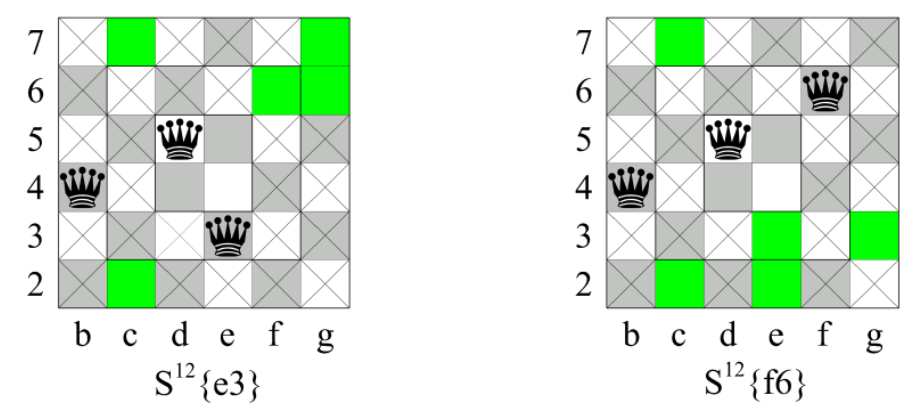

Figure 7: Sub-case 1.2. Graphical representation of two alternatives for placing one queen on subpanel $S^{12}$.

Since b4 is selected by our constraints, two queens must be placed in a6 and c8 squares. No solution is possible because it is not allowed to put four queens on subpanel $\mathrm{S}^{28}$. Figure 8 (left hand) represents this alternative.

- If f6 square is selected on subpanel $S^{12}$, then the squares allowed on subpanel $S^{20}$ are c2, e2, g3 and c7. To satisfy the constraint for placing two queens on subpanel $S^{20}$, the alternatives are $\{\mathrm{b} 4, \mathrm{c} 2\},\{\mathrm{b} 4, \mathrm{e} 2\},\{\mathrm{b} 4, \mathrm{~g} 3\}$ and $\{\mathrm{b} 4, \mathrm{c} 7\}$ squares. Since b4 is selected by our constraints, one queen must be placed in a7 square and this would force to place a queen in c1 square that would force again to place a queen in e8 square. So, $\{\mathrm{b} 4, \mathrm{~g} 3\}$ squares is the only one alternative. No solution is possible because it is not allowed to put four queens on subpanel $\mathrm{S}^{28}$. Figure 8 (right hand) represents this alternative.
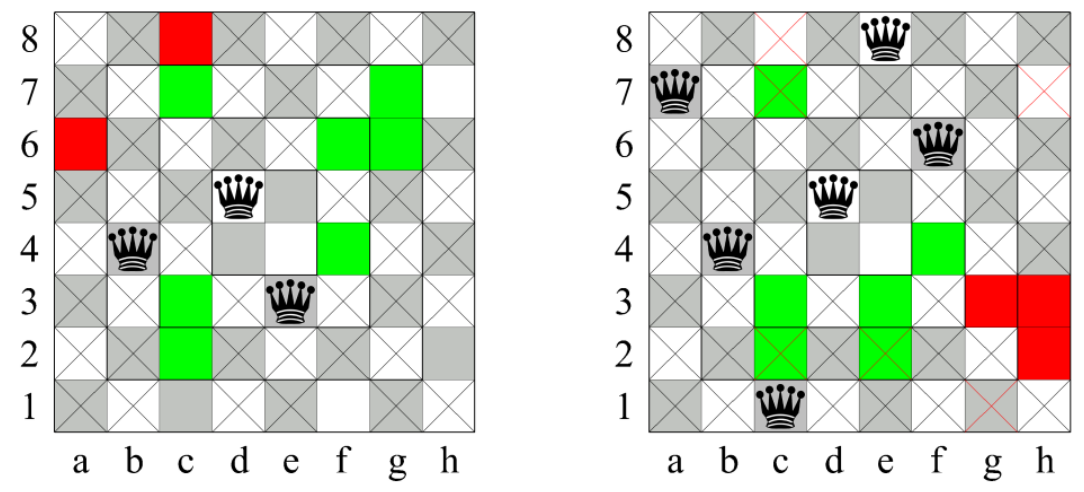

Figure 8: Sub-case 1.2. Graphical representation of two alternatives. Left hand if e3 square is selected and right hand if f6 square is selected on subpanel $S^{12}$.

Sub-case 1.3: There are two queens on subpanel $S^{12}$ and there is one queen on sub- 
panel $S^{20}$. (See Table 2)

The following constraints can be considered:

- There is one queen on subpanel $S^{4}$ and it must be placed in d5 square.

- There are two queens on subpanel $S^{12}$.

- There is one queen on subpanel $S^{20}$ and it must be placed in b4 square.

- There are four queens on subpanel $S^{28}$. So, it is not allowed to place any queen in a1, a8, h1 and h8 squares.

Chessboard reconstruction from subpanel $S^{4}$ is represented in Figure 9. No solution is possible because e3 and $\mathrm{f} 6$ squares allowed on subpanel $S^{12}$ must be selected and it is not allowed to put four queens on subpanel $S^{28}$.

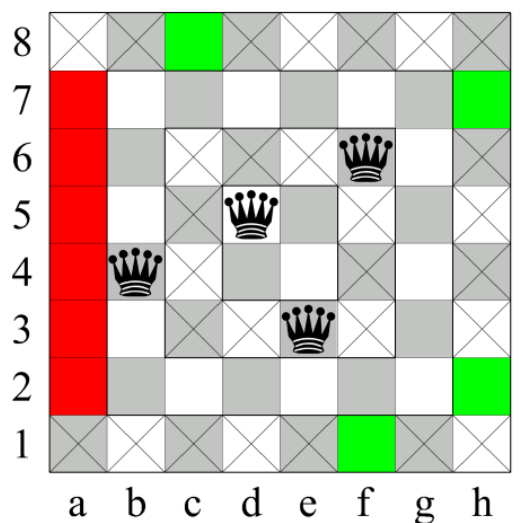

Figure 9: Sub-case 1.3. Graphical representation.

Sub-case 2.1: There is not any queen on subpanel $S^{12}$ and there are four queens on subpanel $S^{20}$. (See Table 2)

The following constraints can be considered:

- There is one queen on subpanel $S^{4}$ and it must be placed in d5 square.

- There is not any queen on subpanel $S^{12}$.

- There are four queens on subpanel $S^{20}$ and one must be placed in b4 square. So, it is not allowed to place any queen in b2, b7, g2 and g7 squares. (g7 is not allowed by the constraint to have exactly 4 queens on $S^{20}$ ). 
- There are three queens on subpanel $S^{28}$. So, at least a queen must be placed in a1, a8, h1 or h8 squares.

Chessboard reconstruction from subpanel $S^{4}$ is represented in Figure 10. The squares allowed on subpanel $S^{20}$ are c2, e2, f2, g3, g6 and c7. To satisfy the constraint for placing four queens on subpanel $S^{20}$, the placing of c7 and g6 already prevents us from finding a solution. Indeed no solution is possible because it is not allowed to put three queens on subpanel $\mathrm{S}^{28}$.

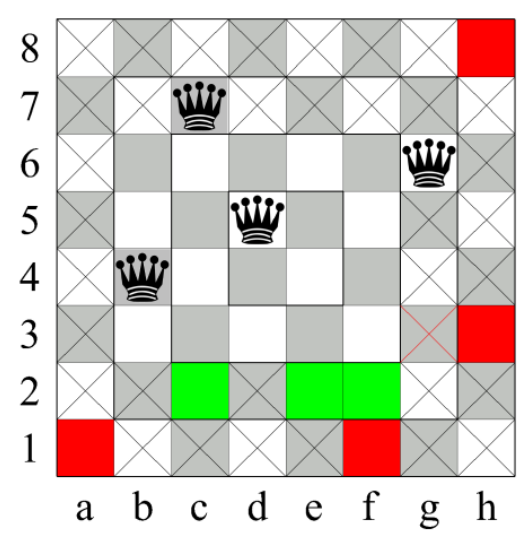

Figure 10: Sub-case 2.1. There is not solution.

Sub-case 2.2: There is one queen on subpanel $S^{12}$ and there are three queens on subpanel $S^{20}$. (See Table 2)

The following constraints can be considered:

- There is one queen on subpanel $S^{4}$ and must be placed in d5 square.

- There is one queen on the subpanel $S^{12}$.

- There are three queens on subpanel $S^{20}$ and one must be placed in b4 square.

- There are three queens on subpanel $S^{28}$. So, at least a queen must be placed in a1, a8, h1 or h8 squares.

Chessboard reconstruction from subpanel $S^{4}$ is represented in Figure 11. The squares allowed on subpanel $S^{12}$ are e3 and f6, and the squares allowed on subpanel $S^{20}$ are c2, e2, f2, g3, g6, g7 and c7.

To satisfy the constraints for placing one queen in the subpanel $S^{12}$, the alternatives are e3 or f6 squares. Figure 12 represents these two alternatives. 


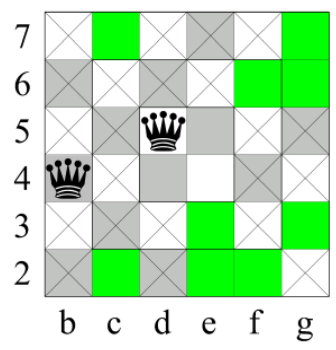

Figure 11: Sub-case 2.2. Squares allowed on sub-panels $\mathrm{S}^{12}$ y $\mathrm{S}^{20}$.
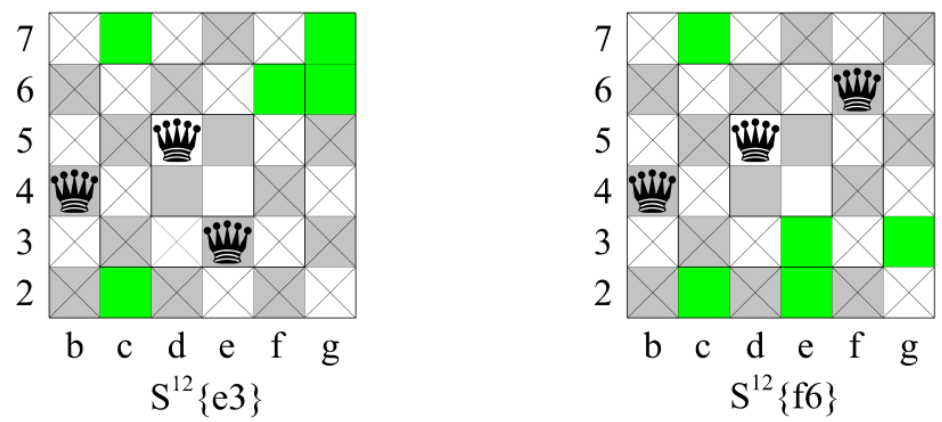

Figure 12: Sub-case 2.2. Graphical representation of subpanel $S^{12}$ alternatives.

- If e3 square is selected on subpanel $S^{12}$, then the squares allowed on subpanel $S^{20}$ are c2, g6, g7 and c7. To satisfy the constraint for placing three queens on subpanel $S^{20}$, the alternatives are $\{\mathrm{b} 4, \mathrm{c} 2, \mathrm{~g} 7\}$ and $\{\mathrm{b} 4, \mathrm{~g} 6, \mathrm{c} 7\}$ squares.

- If $\{\mathrm{b} 4, \mathrm{c} 2, \mathrm{~g} 7\}$ squares are selected then the squares allowed on subpanel $S^{28}$ are f1 and a6. No solution is possible because it is not allowed to put three queens on subpanel $\mathrm{S}^{28}$.

- If $\{\mathrm{b} 4, \mathrm{~g} 6, \mathrm{c} 7\}$ squares are selected then the squares allowed on subpanel $S^{28}$ are $\mathrm{a} 1, \mathrm{f} 1$ and $\mathrm{h} 8$. No solution is possible because it is not allowed to put three queens on subpanel $\mathrm{S}^{28}$.

Figure 13 represents these alternatives.

- If f6 square is selected on subpanel $S^{12}$ then the squares allowed on subpanel $S^{20}$ are $\mathrm{c} 2, \mathrm{e} 2, \mathrm{~g} 3$ and $\mathrm{c} 7$. No solution is possible because it is not allowed to put three queens on subpanel $\mathrm{S}^{28}$. Figure 14 represents this alternative.

Sub-case 2.3. There are two queens on subpanel $S^{12}$ and there are two queens on subpanel $S^{20}$. (See Table 2)

The following constraints can be configured: 

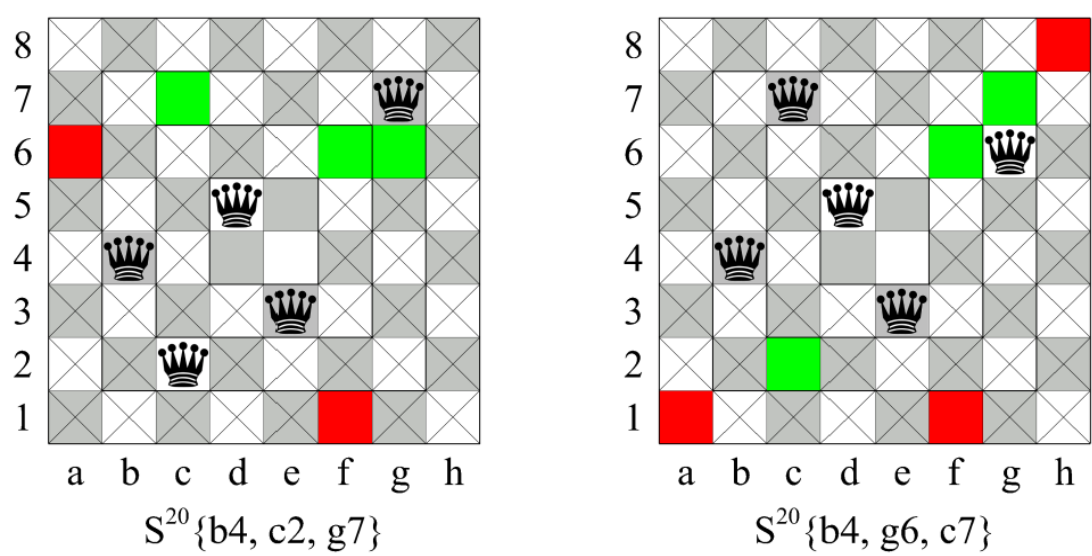

Figure 13: Sub-case 2.2. Graphical representation of subpanel $S^{20}$ and subpanel $S^{28}$ alternatives when e3 square is selected in subpanel $S^{12}$.

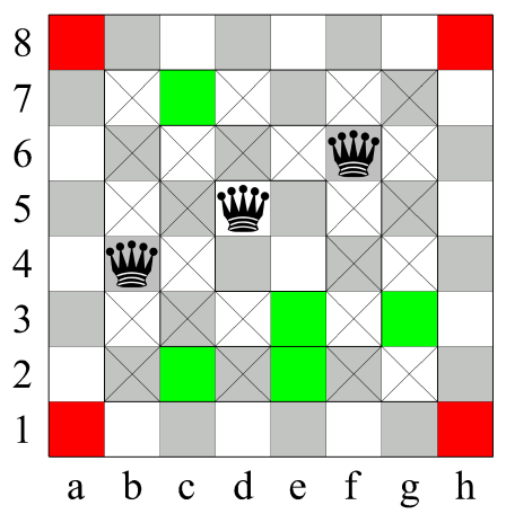

Figure 14: Sub-case 2.2. Graphical representation of subpanel $S^{20}$ and subpanel $S^{28}$ when $\mathrm{f} 6$ square is selected in subpanel $S^{12}$.

- There is one queen on subpanel $S^{4}$ and must be placed in d5 square.

- There are two queens on subpanel $S^{12}$.

- There are two queens on subpanel $S^{20}$ and one must be placed in b4 square.

- There are three queens on subpanel $S^{28}$. So, at least a queen must be placed in a1, a8, h1 or h8 squares.

Chessboard reconstruction from subpanel $S^{4}$ is represented in Figure 15 . The squares allowed on subpanel $S^{12}$ are e3 and f6. The placing of d5 blocks a8 and h1 and the 
mandatory choice of $\mathrm{f} 6$ in $S^{12}$ blocks the other two corners. Indeed no solution is possible because it is not allowed to put three queens on subpanel $S^{28}$.

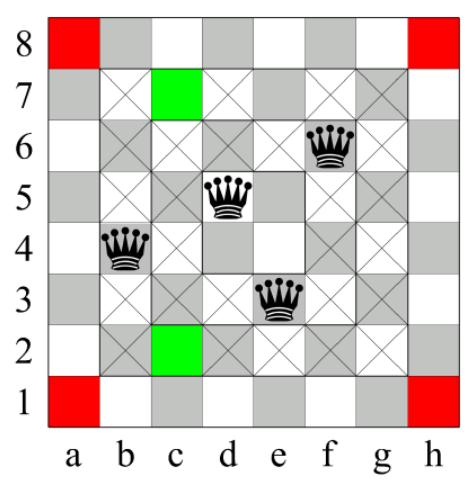

Figure 15: Sub-case 2.3. Graphical representation of subpanel $S^{28}$. It is not allowed to place at least one queen in a1, h1, a8 or h8 squares.

Sub-case 2.4. There are three queens on subpanel $S^{12}$ and there is one queen on subpanel $S^{20}$. (See Table 2)

The following constraints can be considered:

- There is one queen on subpanel $S^{4}$ and must be placed in d5 square.

- There are three queens on subpanel $S^{12}$.

- There is one queen on subpanel $S^{20}$ and must be placed in b4 square.

- There are three queens in the subpanel $S^{28}$. So, at least a queen must be placed in a1, a8, h1 or h8 squares.

Chessboard reconstruction from subpanel $S^{4}$ is represented in Figure 16 . The squares allowed on subpanel $S^{12}$ are c3, e3, $\mathrm{f} 4$ and $\mathrm{f} 6$. No solution is possible because it is not allowed to put three queens on subpanel $\mathrm{S}^{12}$.

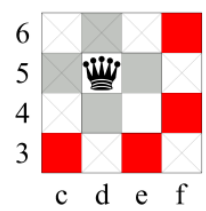

Figure 16: Sub-case 2.4. There is not solution.

Finally, two solutions have been found. They are represented in Figure 17. 

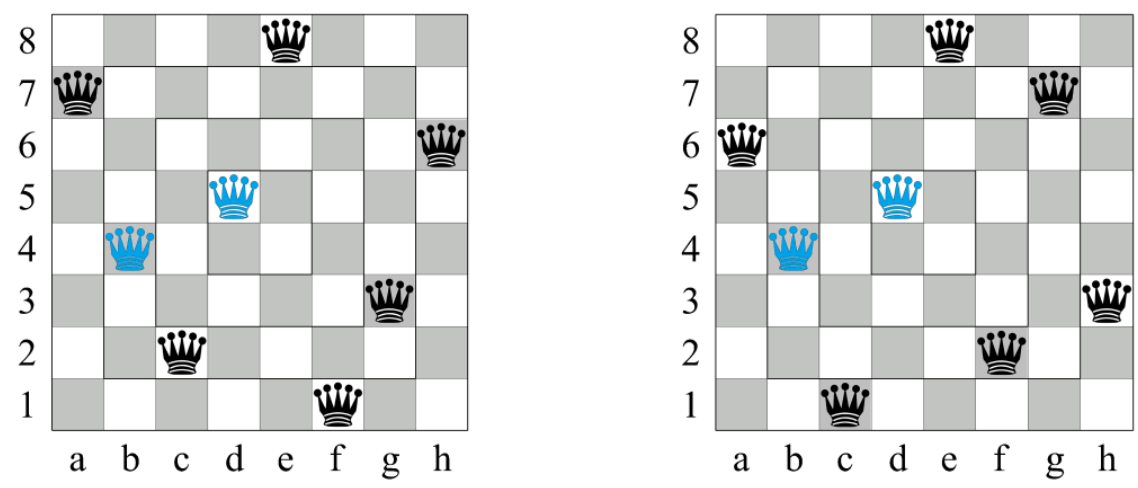

Figure 17: Solutions of the 8-Queens completion puzzle.

\section{References}

[GJN17] Ian P. Gent, Christopher Jefferson and Peter Nightingale. "Complexity of nQueens Completion." Journal of Artificial Intelligence Research Vol. 59 (2017): 815848 DOI: https://doi.org/10.1613/jair.5512

[CMA21] Clay Mathematis Institute. "P vs NP Problem." Accessed 21 September, 2021. https://www.claymath.org/millennium-problems/p-vs-np-problem

[Coo71] Stephen A. Cook. "The complexity of theorem proving procedures." Proceedings Third Annual ACM Symposium on the Theory of Computing, 1971.

[Sch15] Fred Schuh. The Master book of mathematical recreations. Translated by F. Göbel. New York: Dover Publications, 1968: 34-346

[BSR08] Roman Barták, Miguel A. Salido and Francesca Rossi. "Constraint Satisfaction Techniques in Planning and Scheduling." Journal of Intelligent Manufacturing 21, (2010): 5-15 DOI: https://doi.org/10.1007/s10845-008-0203-4.

[BSR10] Roman Barták, Miguel A. Salido and Francesca Rossi. "New Trends in Constraint Satisfaction, Planning, and Scheduling: A Survey." The Knowledge Engineering Review $25 \quad$ (3) (2010): 249-279 DOI: https://doi.org/10.1017/S0269888910000202

[ACP68] Donald E. Knuth. The Art of Computer Programming, Volume 4, Fascicle 5: Mathematical Preliminaries Redux; Introduction to Backtracking; Dancing Links. Addison-Wesley Professional, 2019.

[ACJ18] Nélia Amado, Susana Carreira and Keith Jones. Broadening the Scope of Research on Mathematical Problem Solving. Springer, 2018: 551-552 
[PrE17] Thomas B. Preußer and Mathias R. Engelhardt. "Putting Queens in Carry Chains, Noź27." Journal of Signal Processing Systems, 88(2). (2017):185--201. DOI: https://doi.org/10.1007/s11265-016-1176-8

[AY89] B. Abramson and M. Yung. "Divide and conquer under global constraints: a solution to the $n$-queens probelm," Journal of Parallel and Distributed Computing 6(3), (1989): 649-662

[BS09] J. Bell and B. Stevens. "A survey of known results and research areas for $n$-queens." Discrete Mathematics, 309 (2009): 1--31. DOI: http://dx.doi.org/10.1016/j.disc.2007.12.043

[BD75] A. A. Bruen and R. Dixon. "The n-queen problem." Discrete Mathematics, 12, (1975): 393-395. DOI: https://doi.org/10.1016/0012-365X(75)90079-5

[BM02] A. P. Burger and C. M. Mynhardt. "An upper bound for the minimum number of queens covering the $n \times n$ chessboard." Discrete Applied Mathematics 121, (2002): 51-60. DOI: https://doi.org/10.1016/S0166-218X(01)00244-X

[Eng07] M. R. Engelhardt. "A group-based search for solutions of the n-queens problem." Discrete Mathematics 307, (2007): 2535-2551. DOI: https://doi.org/10.1016/j.disc.2007.01.007

[ET92] C. Erbas, S. Sarkeshik and M. M. Tanik, "Different perspectives of the $N$ queens problem." Proceedings ACM Computer Science Conference (1992): 99--108. DOI: https:/doi.org/10.1145/131214.131227

[Ber91] Bo Bernhardsson. "Explicit Solutions to the N-Queens Problem for All N." ACM SIGART Bulletin 2 (2) (1991). DOI: https://doi.org/10.1145/122319.122322

[GJ79] Michael R. Garey and David S. Johnson. Computers and Intractability: A Guide to the Theory of NP-Completeness. W. H. Freeman, 1979. ISBN 978-0-7167-1045-5

[CMA21] Clay Mathematis Institute. "The 8-Queens Puzzle." Accessed 21 September, 2021. https://www.claymath.org/events/news/8-queens-puzzle 